\title{
COVID-19 Lockdowns: A Worldwide Survey of Circadian Rhythms and Sleep Quality in 3911 Athletes from 49 Countries, with Data-Driven Recommendations
}

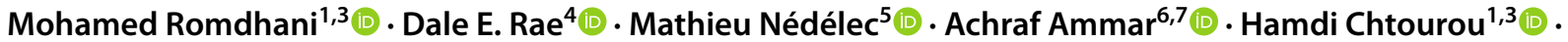

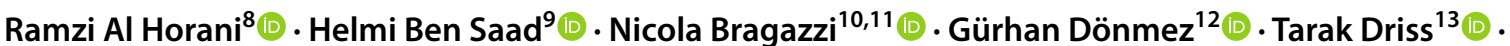

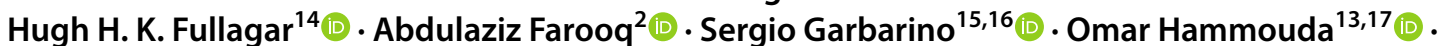

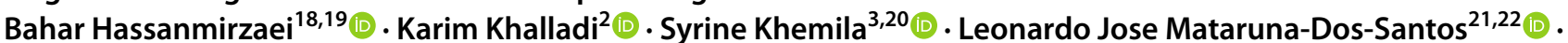

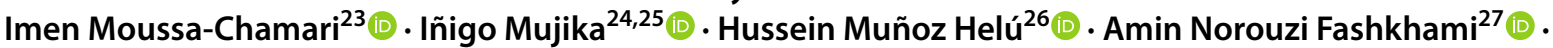

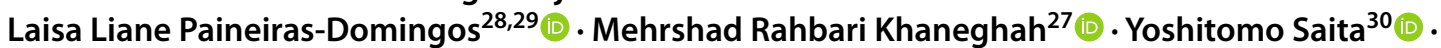 \\ Khaled Trabelsi $i^{1,31}$ ( Jacopo Antonino Vitale ${ }^{32}$ (1) Jad Adrian Washif ${ }^{33}$ (1) . Johanna Weber ${ }^{34,35}$. \\ Nizar Souissi ${ }^{3,20} \cdot$ Lee Taylor $^{36,37} \cdot$ Karim Chamari ${ }^{2,20}$
}

Accepted: 9 November 2021 / Published online: 8 December 2021

(C) The Author(s), under exclusive licence to Springer Nature Switzerland AG 2021

\begin{abstract}
Objective In a convenience sample of athletes, we conducted a survey of COVID-19-mediated lockdown (termed 'lockdown' from this point forward) effects on: (i) circadian rhythms; (ii) sleep; (iii) eating; and (iv) training behaviors.

Methods In total, 3911 athletes [mean age: 25.1 (range 18-61) years, 1764 female (45\%), 2427 team-sport (63\%) and 1442 elite (37\%) athletes] from 49 countries completed a multilingual cross-sectional survey including the Pittsburgh Sleep Quality Index and Insomnia Severity Index questionnaires, alongside bespoke questions about napping, training, and nutrition behaviors.

Results Pittsburgh Sleep Quality Index $(4.3 \pm 2.4$ to $5.8 \pm 3.1)$ and Insomnia Severity Index (4.8 \pm 4.7 to $7.2 \pm 6.4)$ scores increased from pre- to during lockdown $(p<0.001)$. Pittsburgh Sleep Quality Index was predominantly influenced by sleeponset latency $(p<0.001 ;+29.8 \%)$, sleep efficiency $(p<0.001 ;-21.1 \%)$, and total sleep time $(p<0.001 ;-20.1 \%)$, whilst Insomnia Severity Index was affected by sleep-onset latency $(p<0.001 ;+21.4 \%)$, bedtime $(p<0.001 ;+9.4 \%)$, and eating after midnight $(p<0.001 ;+9.1 \%)$. During lockdown, athletes reported fewer training sessions per week $(-29.1 \% ; d=0.99)$. Athletes went to bed ( $+75 \mathrm{~min} ; 5.4 \% ; d=1.14)$ and woke up $(+150 \mathrm{~min} ; 34.5 \% ; d=1.71)$ later during lockdown with an increased total sleep time ( $+48 \mathrm{~min} ; 10.6 \% ; d=0.83)$. Lockdown-mediated circadian disruption had more deleterious effects on the sleep quality of individual-sport athletes compared with team-sport athletes $(p<0.001 ; d=0.41)$, elite compared with non-elite athletes $(p=0.028 ; d=0.44)$ and older compared with younger $(p=0.008 ; d=0.46)$ athletes.

Conclusions These lockdown-induced behavioral changes reduced sleep quality and increased insomnia in athletes. Datadriven and evidence-based recommendations to counter these include, but are not limited to: (i) early outdoor training; (ii) regular meal scheduling (whilst avoiding meals prior to bedtime and caffeine in the evening) with appropriate composition; (iii) regular bedtimes and wake-up times; and (iv) avoidance of long and/or late naps.
\end{abstract}

Lee Taylor and Karim Chamari contributed equally to this manuscript.

Extended author information available on the last page of the article 


\section{Key Points}

Several modifiable behavioral changes were evident within the sleep-COVID-19-lockdown nexus.

Low sleep quality and high insomnia during lockdown were most associated with late bedtimes and long sleeponset latency.

Elite, individual-sports, and older athletes were more vulnerable to these lockdown-induced disruptions compared with non-elite, team sports, and younger athletes, respectively.

Female athletes reported lower sleep quality and higher insomnia compared with male athletes irrespective of the lockdown.

Athletes sleep and circadian rhythms were more affected by lockdown compared with those seen in the general population.

\section{Introduction}

The severe acute respiratory syndrome coronavirus 2-mediated coronavirus disease 2019 (COVID-19) pandemic enforced global change upon day-to-day life [1]. Most nations responded with one or more periods of localised or country-wide lockdowns, resulting in increased anxiety and depression [1,2], sedentary lifestyle, and 'screen time' [3]. Holistically, lockdown-mediated home confinement resulted in lower social participation and life satisfaction, increased psychological strain, social and physical inactivity, an unhealthy diet, and poor sleep hygiene [3-7], particularly among female and younger individuals [8].

Human sleep is regulated by circadian [e.g. zeitgebers (outdoor light, physical activity, meal timing [9])] and homeostatic (e.g. sleep pressure) processes/components, conspiring to produce a consolidated period of nocturnal sleep $[9,10]$. The homeostatic component increases exponentially with the duration and intensity of activity during wakefulness [10]. Exercise can modify subsequent sleep, by increasing sleep pressure [11] and efficiency [12] whilst reducing sleep-onset latency [12]. Regular exercise training, especially outdoors, acts on homeostatic sleep regulation (increasing sleep pressure) and on the circadian component (synchronizing biological rhythms), particularly in athletic populations $[13,14]$. As such, sleep disruption has the potential to impair athletic performance $[15,16]$, whilst the intensity and timing of training could also modify sleep architecture [11, 12, 14, 17, 18]. Compounding this concern, athletes are purported to require greater sleep (than non-athletes) to promote adequate/optimal physical, physiological, and psychological recovery/regeneration [19, 20]. Despite this and as reviewed extensively [19-22], it is common for athletes to: (i) sleep less than recommended values (i.e. 7-9 h for healthy adults [23]); (ii) self-report low perceived sleep quality; and (iii) demonstrate a high prevalence of insomnia-like symptoms (e.g., non-restorative sleep, difficulties in initiating and maintaining sleep [19, 22]).

In athletes, in part because of lockdowns, reduced sleep quality and frequency of training exposures were associated with reduced mental health $[6,7]$. Sleep is critical to athletes for recovery and is well reported as their first and preferred recuperation strategy [24]. Indeed, athletes usually increase their sleep duration on 'off-days' (i.e., when they do not have to train or compete [24]), likely a reflection that many are chronically sleep deprived [20,21]. Athletes increased the time spent in bed during lockdown [6], whilst going to bed and waking later compared with pre-lockdown [6, 7], indicative of a chronobiological and circadian disruption [6]. This desynchrony can alter the temporality and composition of dietary intake alongside undesirable changes in metabolism and immunity [25]; the latter of particular concern during a pandemic [26]. These lockdown-induced behavioural changes have been shown to be detrimental to athletes' mental health (e.g., depression) and physical capacities (e.g., deconditioning), likely disrupting their return to training and competition post-COVID-19 [6, 7].

In light of previous findings in the general population [8] and in athletes [6,7], and given the importance of sleep for the performance and health of athletes, the lockdown could plausibly have more pronounced effects on the sleep of athletic populations compared with non-athletic populations. Thus, it appears prudent to survey and understand the effects of lockdown on sleep within athletic populations. Therefore, the present study aimed to survey COVID-19-mediated behavioral changes (comparing pre-lockdown to within lockdown) in: (i) circadian rhythms; (ii) sleep patterns; (iii) eating behaviors; and (iv) training habits in athletes who had experienced a period of lockdown. A secondary aim was to determine specific modifiable factors related to any apparent increased insomnia and lowered sleep quality during lockdown, in order to develop data-driven and evidence-based recommendations for future lockdown-like situations. It was hypothesized that: (1) the lockdown will negatively influence the measured sleep parameters within athletes and to a greater magnitude than that seen in non-athletes; (2) the change in sleep, training, and eating behaviors will affect sleep quality and insomnia in athletes; and (3) such undesired effects will be more pronounced in: elite vs non-elite athletes; younger vs older athletes; and female vs male athletes. 


\section{Procedure}

Informed consent was provided by participants under ethical approval from Farhat HACHED Hospital, Sousse, Tunisia (IRB\# FH020720) in the spirit of the Declaration of Helsinki. Data were processed anonymously and according to the guidelines of the "General Data Protection Regulation" (gdpr-info.eu). A priori sample size estimation was 3710 (see Electronic Supplementary Material [ESM] for full details) according to Whitley and Ball [27], as per the literature evidence [7]. The assumption of $12 \%$ for duplicate participants, entry errors, and eligibility of inclusion/ exclusion criteria gave a revised sample of 4218 participants $[4218=3710 /(1.0-0.12)]$.

A cross-sectional, global, and web-based questionnaire was used, developed by 32 multidisciplinary scientists and academics (research team) from 39 research centers. It surveyed: (i) circadian rhythms; (ii) sleep patterns; (iii) eating behaviors; and (iv) training habits in athletes who had experienced a period of lockdown. At survey launch (and when participants completed their responses) and reflective of the global pandemic landscape, countries had begun or began (within an 'open' survey window) their 'first' lockdowns, whilst some had had their lockdown tempered or lifted. Consequently, the month preceding each respondent's lockdown 'start' was utilized as their individual baseline. Thus, the present comparisons are made by asking participants to compare the 'month preceding lockdown' (i.e., baseline/normality; relying on their recall of activities during this time) to 'during lockdown' (in some participants, this relied on recall as their lockdown had been tempered or lifted). The original English survey was translated into nine languages (Arabic, French, Italian, Japanese, Malay, Persian, Portuguese, Spanish, and Turkish). The survey questions underwent translation and back-translation, performed by the research team (including at least one native speaker and one topic expert). Thereafter, $1 \%$ of the participants (42 athletes) responded to the questionnaire twice (1 week apart), assessing reliability. The survey was launched online through social media (e.g., Facebook, Twitter, WhatsApp, and e-mail), opening 8 July and closing 30 September, 2020 (all survey responses were received within this window with $37 \%$ of the participants still in lockdown when providing survey responses), after reaching the required sample size.

\subsection{Participants and Reliability}

Eligibility criteria for participants were: (i) $\geq 18$ years of age; (ii) classified as an athlete (competing at any given level; individual or team sport); and (iii) had experienced a period of lockdown for at least 2 weeks. For the purposes of who met the criteria of being an 'athlete', the survey preamble explicitly stated "competing at any given level". For analysis purposes, subgroups were created: sex (male vs female); competitive level (elite vs non-elite); sport discipline (individual vs team sport); and age groups [18-24 (younger) and $\geq 25$ (older) years [28]]. The adopted age subgroups reflect the World Health Organization classifications of 'Adolescents (10-19 years),' 'Youth (15-24 years),' and 'Young People (10-24 years)' [28]. As our 'younger' subgroup captures only 2 years of late adolescence it was more appropriate, and satisfied statistical power requirements, to use the World Health Organization classification to guide the maximum age (24 years) of our 'younger' group, whilst the minimum age was 18 years to satisfy ethical approval requirements. See the ESM (part I; question 6) for descriptive criteria regarding how participant 'competitive level' was determined. Specifically, participants classed as 'elite' and 'professional' from the survey were collapsed into the elite subgroup, within the non-elite subgroup all remaining competitive level categories were collapsed.

\subsection{Survey}

The survey had six sections and the full English version can be found within the ESM. Section 1: Explanation of the study; invited volunteers to confirm eligibility; provided consent to participate; and encouraged respondents to respond as accurately as possible. Section 2: Demographic and lockdown questions (e.g., sex, age, country of residence, the lockdown duration, the sport discipline, and the level of competition). Section 3: Training questions: Athletes were asked about their preferred time of day (TOD) to train and the number of training sessions per week. Section 4: PSQI: The PSQI was used to assess subjective sleep quality. The global PSQI score ranges from 0 to 21 with lower scores indicating better sleep quality. A PSQI score of $\geq 5$ indicates a poor sleep quality and a score of $\geq 8$ indicates highly disturbed sleep [29]. Self-reported bedtimes and wake-up times, total sleep time, time in bed, sleep efficiency, and sleep-onset latency were also derived from the PSQI (explained in Table 1). ISI: The ISI consists of seven items rating insomnia (0-4 Likert scale), with the summed total (between 0 and 28) indicating moderate (15-21) or severe (22-28) clinical insomnia [30]. Section 5: Napping questions: Athletes were asked about their napping behavior (e.g., nap timing, duration, and frequency). Section 6: Nutrition-related and health-related questions: Nutrition-related questions included self-reported body mass $(\mathrm{kg})$, number of daily meals eaten, and the likelihood to eat after midnight (very likely 2 ; likely 1 ; unlikely-1; and very unlikely-2) pre- and during lockdown. Furthermore, athletes were asked how many caffeinated and alcoholic beverages they consumed and how many cigarettes they smoked per day pre- and during lockdown. 
Table 1 Definition of different sleep metrics measured in the current study

\begin{tabular}{ll}
\hline Parameters & Definition \\
\hline Bedtime & The time when the athlete goes to bed \\
Sleep Onset Latency (SOL) & The time that separates bedtime (lights off) from the actual start of sleep \\
Wake-up & The time when the athlete gets out of bed \\
Total sleep time (TST) & The time spent asleep, i.e., between sleep onset and wake-up time \\
Time in bed (TIB) & The total time spent in bed, between bedtime and wake-up time \\
Sleep efficiency (SE) & $(\mathrm{TST} / \mathrm{TIB}) \times 100$
\end{tabular}

\subsection{Statistical Analyses}

Statistical analysis was performed using SPSS (SPSS Statistics for Windows, Version 26; IBM Corporation, Armonk, NY, USA) and figures were created using GraphPad Prism 8 (GraphPad Software, San Diego, CA, USA). The effect of lockdown (pre vs during) was assessed using a paired sample t-test. Lockdown's effects on (i) circadian rhythms, (ii) sleep, (iii) eating; and (iv) training behaviors between sub-groups of sex (male vs female), competitive level (nonelite vs elite), sport discipline (team vs individual), and age (younger vs older) were analyzed using a multivariate, repeated-measures analysis of variance (ANOVA). Significant effects underwent a Bonferroni post-hoc test with the Cohen's effect size $(d)$ subsequently calculated, qualitatively interpreted as small $(d<0.5)$, moderate $(0.5 \leq d<0.8)$, and large $(d \geq 0.8)$ [31].

Multiple linear regression analyses described the relationships between dependent (PSQI and ISI scores) and independent (sleep, training, health, and nutrition-related) variables. The multiple regression model was based on delta variation $(\Delta \% ; \%$ change in each variable pre- to during lockdown) according to the formula [100 $\times$ (during lockdown - pre-lockdown)/pre-lockdown]. All the independent variables were entered into the model using a stepwise method. We report the $R$ square $\left(R^{2}\right.$; the proportion of variance in the dependent variable explained by independent variables) for the entire model as well as the semi-partial correlation coefficient squared $\left(s r^{2}\right)$ to indicate the unique contribution (in percentage) of each independent variable within the model. The multiple regression model was completed by further statistical mediation to quantify the indirect effect of independent variables on the dependent variables (i.e., PSQI and ISI) according to Hayes' guidelines [32]. The magnitude of the mediation effect is expressed as a percentage according to the formula (indirect effect/total effect $x$ 100). All values within the text and tables are reported as mean \pm standard deviation, and as $95 \%$ confidence intervals in the figures. Alpha was set at $p<0.05$.

Comparison between athletes (present study) and general population [5] data were made by entering pre- and during lockdown replicate data (mean, standard deviation, and number of participants) into two-way ANOVA (pre-/during lockdown * athletes/general population), followed by Bonferroni Post-hoc test, using GraphPad software.

\section{Results}

\subsection{Participants' Characteristics}

A total of 4218 survey responses were received. After removing duplicates, incomplete data, and invalid responses, 3911 valid responses were used for analysis (mean age: $25.06 \pm 8.9$; range $18-61$ years) from 49 countries, continentally located as: Asia $(57 \% ; n=2237)$; North and South America (18\%; $n=720)$; Europe $(14 \% n=534)$; Africa $(11 \%$ $n=414)$; and Australasia $(0.1 \% n=6)$. The sample represents 56 sport disciplines; team (63\%) and individual (37\%) sport athletes, competing at elite (37\%) and non-elite (63\%) levels; with 54\% $(n=2106)$ male and $45 \%(n=1764)$ female [1\% $(n=41)$ preferring not to declare their sex, subsequently removed to facilitate binary sex analysis] distributed as per the following regarding age: younger $(67 \% ; n=2595)$ and older $(33 \% ; n=1275)$.

\subsection{Univariate Analysis}

Overall PSQI and ISI scores were higher during (PSQI: $5.84 \pm 3.16$; ISI: $7.19 \pm 6.41$ ) compared with pre- (PSQI: $4.32 \pm 2.48$; ISI: $4.85 \pm 4.72$ ) lockdown (all $p<0.001$ ). Pre-lockdown, 39\% ( $n=1525)$ of athletes were classified as poor sleepers increasing to $49 \%(n=1917)$ during lockdown $(p<0.001)$. Additionally, $10 \%(n=391)$ of athletes reported highly disrupted sleep pre-lockdown, increasing to $22 \%(n=860)$ during lockdown $(p<0.001)$. Pre-lockdown, $5 \%$ of athletes reported moderate $(4 \%, n=156)$ or severe $(\sim 1 \%, n=23)$ clinical insomnia, increasing to $15 \%$ divided into moderate $(12 \%, n=469)$ and severe $(3 \%, n=117)$ clinical insomnia during lockdown (all $p<0.001$ ). Table 2 summarizes the statistical changes of the primary univariate analyses (sleep, training, nutrition, and health habits) from pre- and during lockdown. 
Table 2 Statistical parameters relating to changes in sleep, training, and nutrition-related and health-related behaviors in response to lockdown

\begin{tabular}{|c|c|c|c|c|c|c|c|c|}
\hline \multirow[t]{2}{*}{ Variable } & \multicolumn{2}{|l|}{ Lockdown } & \multirow[t]{2}{*}{$t$} & \multirow[t]{2}{*}{$N$} & \multirow[t]{2}{*}{$P$-value } & \multirow[t]{2}{*}{$d$} & \multirow[t]{2}{*}{ MD } & \multirow[t]{2}{*}{$95 \% \mathrm{CI}$} \\
\hline & Pre & During & & & & & & \\
\hline PSQI score (au) & $4.33 \pm 2.49$ & $5.85 \pm 3.16$ & 31.41 & 3911 & $<0.001$ & 0.73 & 1.51 & $1.42-1.61$ \\
\hline ISI score (au) & $4.86 \pm 4.73$ & $7.2 \pm 6.41$ & 27.46 & 3911 & $<0.001$ & 0.58 & 2.33 & $2.17-2.51$ \\
\hline Bedtime (hh:mm) & $23: 24 \pm 1: 37$ & $00: 39 \pm 2: 08$ & 46.28 & 3911 & $<0.001$ & 1.11 & $1: 15$ & $72-81$ \\
\hline Wake-up time (hh:mm) & $7: 15 \pm 1: 38$ & $9: 45 \pm 2: 27$ & 65.98 & 3911 & $<0.001$ & 1.71 & $2: 30$ & $145-154$ \\
\hline Total sleep time (min) & $444 \pm 69$ & $492 \pm 87$ & 35.09 & 3911 & $<0.001$ & 0.86 & 48 & $45-50$ \\
\hline Time in bed (min) & $498 \pm 89$ & $588 \pm 110$ & 50.85 & 3911 & $<0.001$ & 0.89 & 90 & $86-93$ \\
\hline Sleep efficiency $(\%)$ & $90.24 \pm 12.9$ & $84.98 \pm 22.1$ & 13.78 & 3911 & $<0.001$ & 0.41 & -5.28 & $4.53-6.03$ \\
\hline Sleep-onset latency (min) & $21.1 \pm 16.33$ & $35.5 \pm 29.51$ & 34.81 & 3911 & $<0.001$ & 0.86 & 14 & $13-15$ \\
\hline Nap frequency $\left(\mathrm{N}^{\circ} \cdot\right.$ week $\left.^{-1}\right)$ & $1.72 \pm 1.64$ & $2.1 \pm 1.78$ & 12.31 & 3560 & $<0.001$ & 0.31 & 0.41 & $0.35-0.48$ \\
\hline Nap duration (min) & $14.21 \pm 20.6$ & $21.1 \pm 25.46$ & 16.31 & 3911 & $<0.001$ & 0.41 & 7 & $6-7$ \\
\hline Nap timing (hh: mm) & $14: 33 \pm 1: 54$ & $14: 46 \pm 1: 59$ & 5.92 & 2542 & $<0.001$ & 0.16 & $0: 11$ & $7-14$ \\
\hline 24-h sleep duration (min) & $459 \pm 72$ & $514 \pm 92$ & 37.94 & 3911 & $<0.001$ & 0.93 & 55 & $52-57$ \\
\hline Preferred training TOD (hh: mm) & $14: 20 \pm 4: 33$ & $14: 45 \pm 5: 51$ & 4.55 & 3911 & $<0.001$ & 0.11 & $0: 20$ & $11-29$ \\
\hline Training sessions $\left(\mathrm{N}^{\circ} \cdot\right.$ week $\left.^{-1}\right)$ & $3.34 \pm 2.32$ & $4.96 \pm 2.32$ & 41.19 & 3903 & $<0.001$ & 1.01 & -1.41 & $1.34-1.48$ \\
\hline Body mass (kg) & $67.7 \pm 14.62$ & $68.76 \pm 16.3$ & 6.33 & 3908 & $<0.001$ & 0.08 & 1.14 & $0.69-1.49$ \\
\hline Meals $\left(N^{\circ} \cdot\right.$ day $\left.^{-1}\right)$ & $3.06 \pm 1.23$ & $3.09 \pm 1.35$ & 2.46 & 3911 & $<0.05$ & 0.06 & 0.05 & $0.01-0.09$ \\
\hline Eat after midnight $(\mathrm{au})$ & $0.6 \pm 1.57$ & $1.06 \pm 1.33$ & 18.95 & 3668 & $<0.001$ & 0.44 & 0.46 & $0.41-0.51$ \\
\hline Caffeinated beverages $\left(\mathrm{N}^{\circ} \cdot\right.$ day $\left.^{-1}\right)$ & $1.45 \pm 1.6$ & $1.54 \pm 1.66$ & 4.86 & 3694 & $<0.001$ & 0.08 & 0.11 & $0.06-0.14$ \\
\hline Alcoholic beverages $\left(\mathrm{N}^{\circ} \cdot \mathrm{day}^{-1}\right)$ & $0.38 \pm 1.01$ & $0.45 \pm 1.19$ & 4.31 & 3705 & $<0.001$ & 0.08 & 0.06 & $0.03-0.09$ \\
\hline Cigarettes smoked $\left(\mathrm{N}^{\circ} \cdot\right.$ day $\left.^{-1}\right)$ & $1.02 \pm 3.89$ & $1.14 \pm 4.35$ & 2.26 & 3698 & $<0.05$ & 0.03 & 0.09 & $0.01-0.18$ \\
\hline
\end{tabular}

Dependent $t$-tests were used to compare variables measured pre- and during lockdown $a u$ arbitrary unit, $C I$ confidence interval, $d$ Cohen's effect size, $h$ hour, $I S I$ Insomnia Severity Index, $M D$ mean difference, $m i n$ minutes, $N^{\circ}$ number, PSQI Pittsburgh Sleep Quality Index, TOD time of day

\subsection{Multiple Regression}

Multiple linear regression models for PSQI $\left(F_{(10,3900)}=164.15 ; p<0.001 ; R^{2}=0.29\right)$ and ISI $\left(F_{(12,3898)}=50.51 ; p<0.001 ; R^{2}=0.13\right)$ were significant. Both sleep quality and insomnia were mostly affected by the increase of sleep-onset latency and the delayed bedtime (all $p<0.001$ ). Furthermore, the increase in caffeine and alcohol consumption during the lockdown affected sleep quality $(p=0.038, p<0.001)$ and insomnia $(p=0.002, p=0.007)$, respectively. The delayed preferred TOD to train was associated with a lower sleep quality and higher insomnia (both; $p=0.005$ ). However, the reduced number of training sessions per week affected only insomnia scores $(p=0.013)$. Figure 1 presents the results of the multiple regression models.

The change in PSQI was mostly associated with the changes in sleep-onset latency and sleep efficiency, which were in turn mediated by other variables (Fig. 2). Similarly, Fig. 3 presents the variables that mediated the association between sleep-onset latency and bedtime (i.e., independent variables) and ISI (dependent variable).

\subsection{Multivariate Analysis}

Please see ESM for the main effects (i.e., lockdown; competitive level; sport discipline; age; and sex) and interaction (i.e., lockdown $\times$ competitive level; lockdown $\times$ sport discipline; lockdown $\times$ age; and lockdown $\times$ sex) ANOVA output.

\subsubsection{Effect of Competitive Level}

Pre-lockdown, non-elite athletes reported higher PSQI scores compared with elite athletes $(p<0.001)$, with lockdown equalizing this difference, indicative of increased PSQI among elite athletes during lockdown (Fig. 4A). Additionally, elite athletes showed higher insomnia during lockdown compared with non-elite athletes (Fig. 4B). Both sub-groups were napping more frequently and for a longer duration during lockdown compared with pre-lockdown (both; $p<0.001$ ), but elite athletes were napping later during lockdown compared with non-elite athletes $(p=0.003)$ and compared with pre-lockdown $(p<0.001)$. Non-elite athletes were consuming more alcoholic beverages compared 


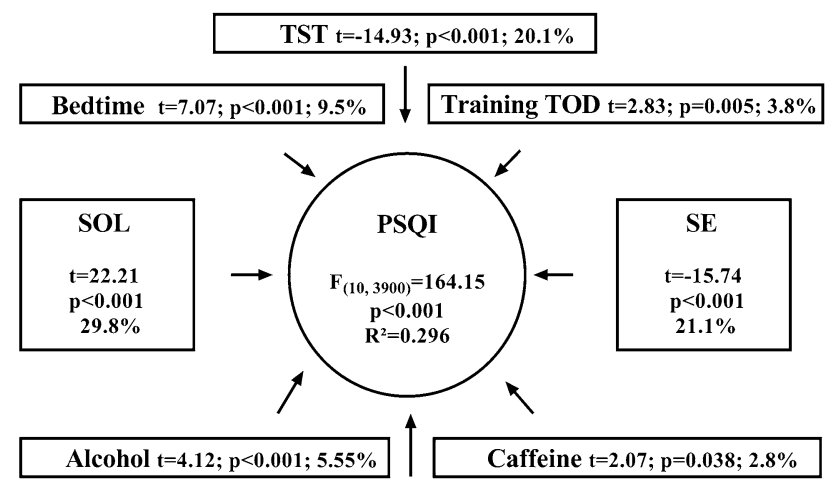

Body mass $\mathrm{t}=2.54 ; \mathrm{p}=0.011 ; 3.4 \%$

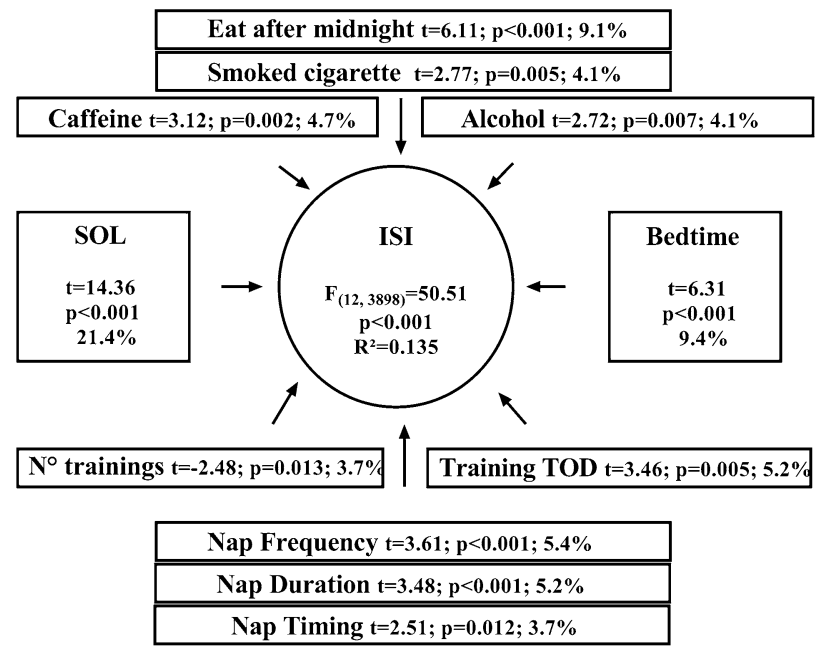

able explained by all the independent variables; \%: semi-partial correlation coefficient squared; indicates the unique contribution of each independent variable within the model; SOL: sleep onset latency; SE: sleep efficiency; TST: total sleep time; TOD: time of day

lockdown, older athletes were napping longer and more frequently compared with younger athletes (both; $p<0.001$ ). Older athletes were napping later pre- $(p=0.006)$ and during $(p<0.001)$ lockdown compared with younger athletes. Younger athletes were consuming more alcoholic and caffeinated beverages and smoking more cigarettes compared with older athletes both during and pre-lockdown (all; $p<00.001)$. Younger athletes were eating more meals prelockdown $(p=0.048)$ and this difference disappeared during lockdown, and the older group reported a higher food consumption after midnight compared with pre-lockdown $(p<0.001)$, with no age-based difference in body mass. Figure 7 presents training behavior and sleep metrics according to the age of athletes.

\subsubsection{Effect of Sex}

Female athletes reported higher PSQI and ISI scores regardless of the lockdown (all; $p<0.001$ ). Napping frequency and duration increased in both sub-groups (all; $p<0.001$ ) and there was no sex-based difference in this increase. However, only female athletes reported a later napping TOD during lockdown compared with pre-lockdown $(p=0.008)$. Male athletes were consuming more alcoholic beverages both during $(p=0.009)$ and pre-lockdown $(p=0.004)$ compared with female athletes, smoking more cigarettes during and prelockdown (both; $p<0.001$ ), with no sex-based difference in caffeine consumption. Male athletes were eating more meals compared with female athletes pre-lockdown $(p=0.015)$ and this difference disappeared during lockdown. Furthermore, male athletes reported a higher likelihood of consuming
Older athletes showed a higher lockdown-induced increase in PSQI and ISI scores (both; $p<0.001$ ) compared with younger athletes (Fig. 4). Pre-lockdown, both groups were napping with the same frequency. However, during 


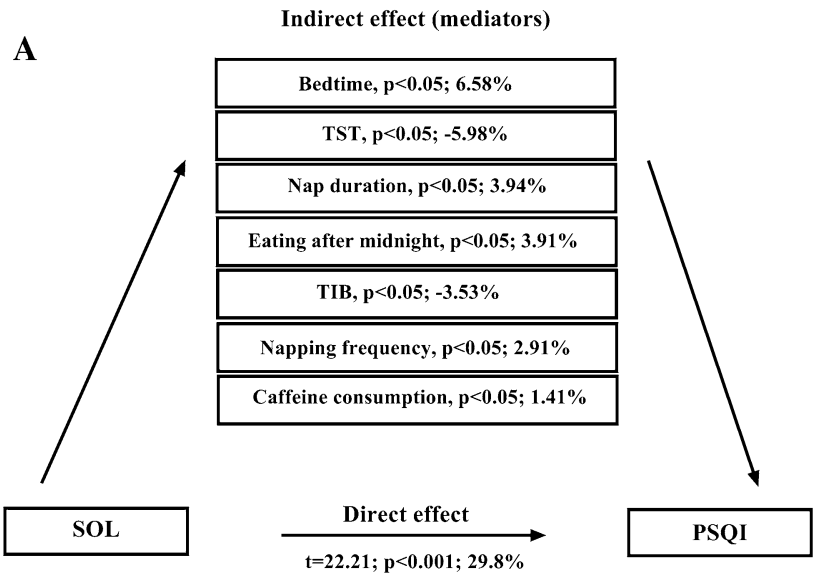

Fig. 2 Left panel (A) indirect mediators affecting the relationship between SOL (sleep onset latency) and PSQI (Pittsburgh sleep quality index). Right panel (B); indirect mediators affecting the relation-

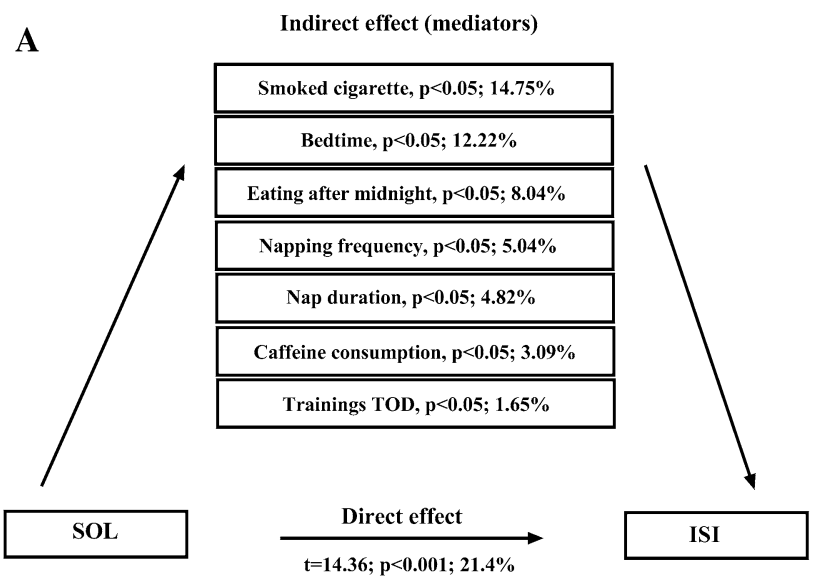

Fig. 3 Left panel (A) indirect mediators affecting the relationship between SOL (sleep onset latency) and ISI (insomnia severity index). Right panel (B); indirect mediators affecting the relationship between

food after midnight compared with female athletes during lockdown $(p=0.001)$ and pre-lockdown $(p=0.005)$, with no sex-based difference in body mass. Training behavior and sleep metrics according to the sex of athletes are presented in Fig. 8.

\section{Discussion}

A phase delay in athletes' circadian rhythms, as measured by sleep timing and training TOD, was seen as a consequence of lockdown. Specifically, lockdown influenced all measured sleep parameters in athletes and to a greater extent compared with previously published non-athlete data [5] [accepting hypothesis (1)]. The lockdown-induced behavioral changes

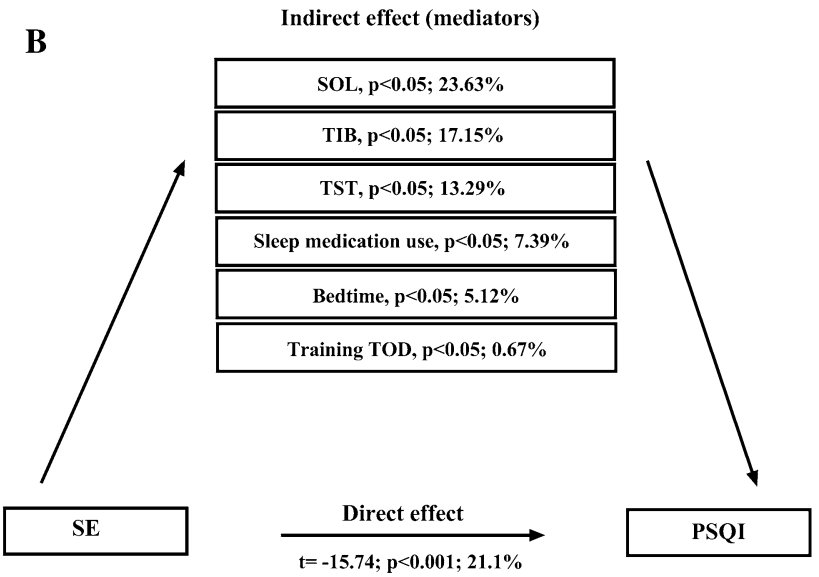

ship between SE (sleep efficiency) and PSQI; TIB: time in bed; TOD: time of day; and TST: total sleep time; \%: the magnitude of the mediation effect

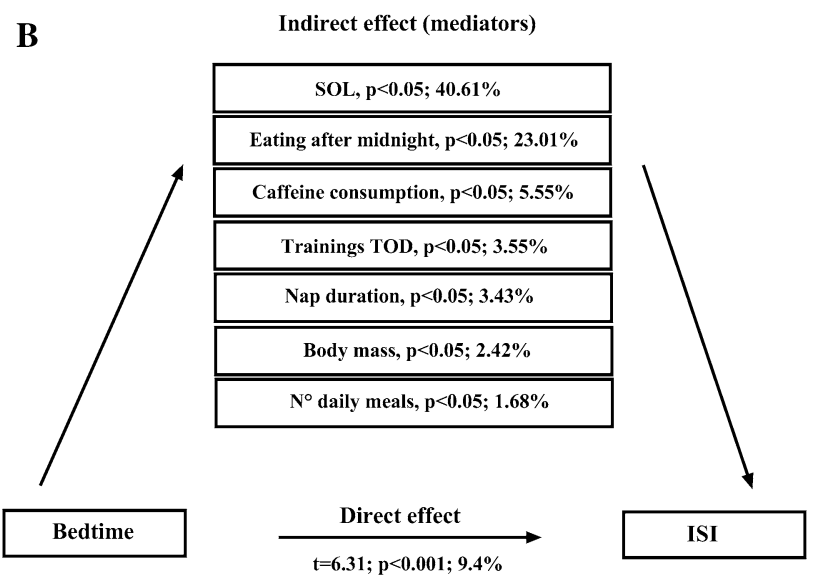

bedtime and ISI; $\mathrm{N}^{\circ}$ : number; TOD; time of day; \%: the magnitude of the mediation effect

(eating, training, and sleep habits) are implicated in the observed reduced sleep quality and increased insomnia [accepting hypothesis (2)]. Lower sleep quality and higher insomnia were mostly associated with longer sleep-onset latency and a later bedtime. Elite athletes demonstrated greater circadian misalignment during lockdown alongside higher insomnia and lower sleep quality compared with nonelite athletes. Female athletes reported a lower sleep quality and higher insomnia-like symptoms compared with male athletes irrespective of lockdown, whilst older athletes were more susceptible to negative and greater magnitude of the lockdown-mediated sleep quality parameter reductions compared with younger athletes (partially accepting our third hypothesis). 

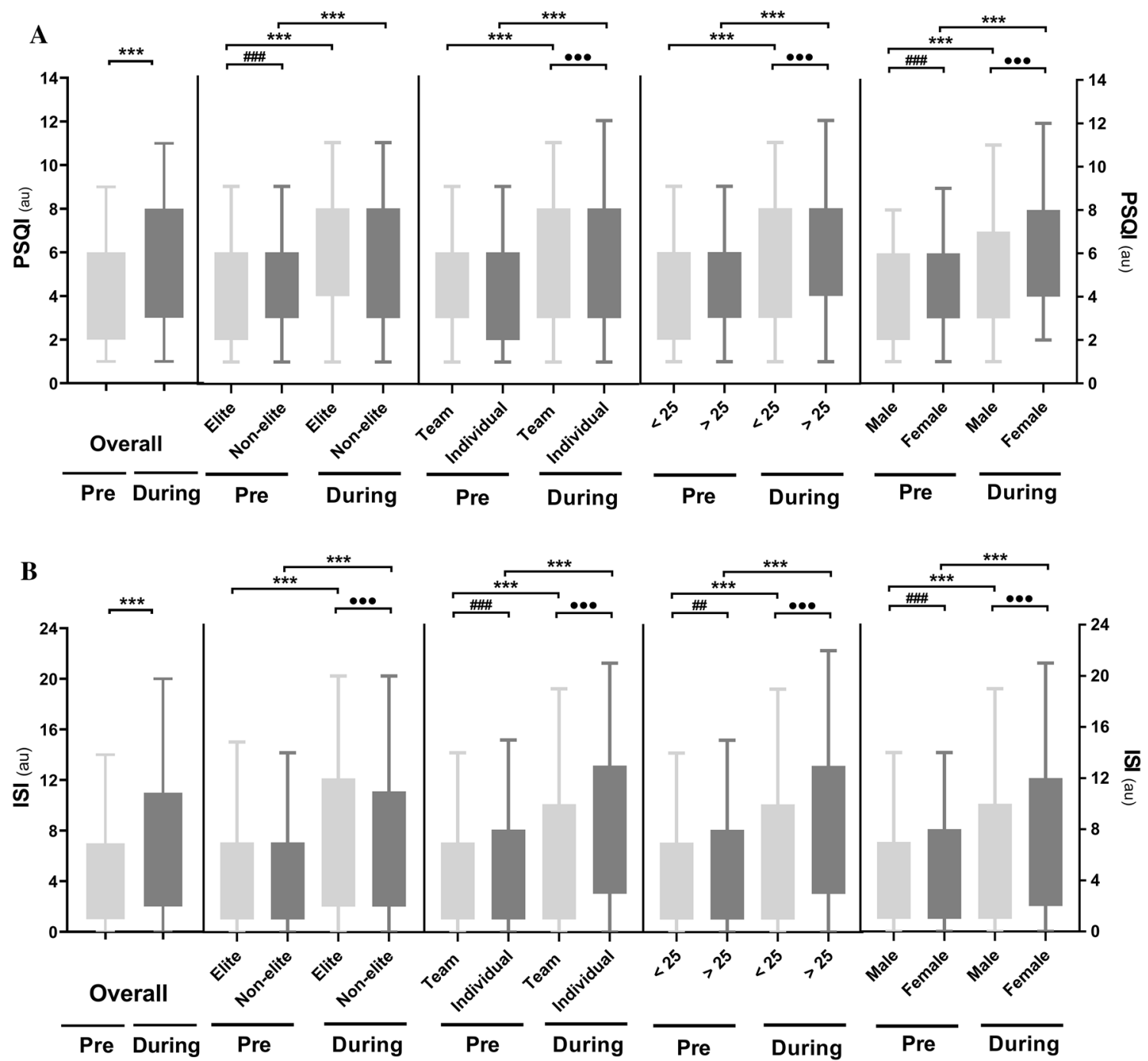

Fig. 4 Mean and 95\% confidence interval for A Pittsburgh Sleep Quality Index (PSQI) and B Insomnia Severity Index (ISI) scores pre- and during lockdown for the overall sample $(n=3911)$ and for subgroups; elite $(n=1442)$ and non-elite $(n=2428)$ athletes; individual-sport $(n=1443)$ and team-sport $(n=2427)$ athletes; age $\leq 25$ years $(n=2595)$ and age $>25$ years $(n=1275)$; male $(n=2106)$ and female $(n=1764)$ athletes. $*, * *$, and $* * *$ mean signiicant effect of the lock-

\subsection{Univariate Effect of the Lockdown}

Athletes showed a greater increase in PSQI scores (35.2\%) owing to the pandemic compared with the general population $(21.7 \%)$ [5]. Indeed, lockdown appears to have affected athletes more than the general public, with greater lockdown-mediated increases in sleep-onset latency [68.6\% (present data) vs $38.2 \%$ (general public data, reported in [5])], time in bed $(18.1 \%$ vs $6 \%)$, and total sleep time $(10.6 \%$ vs $5.7 \%$ ). Surveyed athletes reported 7 h $24 \mathrm{~min} \pm 1$ h $10 \mathrm{~min}$ of sleep pre-lockdown, in line with what has been published in a recent review (athletes slept $7 \mathrm{~h} 20 \mathrm{~min} \pm 1 \mathrm{~h} 10 \mathrm{~min}$ down within groups at $p<0.05, p<0.01$, and $p<0.001$, respectively. Black dots $\cdot, \cdot$, and $\cdots$ mean diference between groups during lockdown at $p<0.05, p<0.01$, and $p<0.001$, respectively. \#, \#, and ${ }^{\# \# \#}$ mean diference between groups pre-lockdown at $p<0.05, p<$ 0.01 , and $p<0.001$, respectively. Signicance is assessed by the twoway analysis of variance and the Bonferroni post-hoc test

per night [33]). Although $7 \mathrm{~h} 24$ min of sleep is within the National Sleep Foundations recommendations for healthy adults (7-9 h [23]), athletes are purported to have a greater sleep need than the general population $[19,20]$. Longer sleep durations during lockdown were associated with lower PSQI scores (i.e., better sleep quality). This may be indicative of insufficient sleep duration pre-lockdown, and when the opportunity for extra sleep is possible, athletes extended their sleep duration [24]. Indeed, sleep extension in athletes has been reported to be beneficial for athletic performance [20], especially after sleep deprivation [16]. However, the longer total sleep time and time in bed negatively mediated 

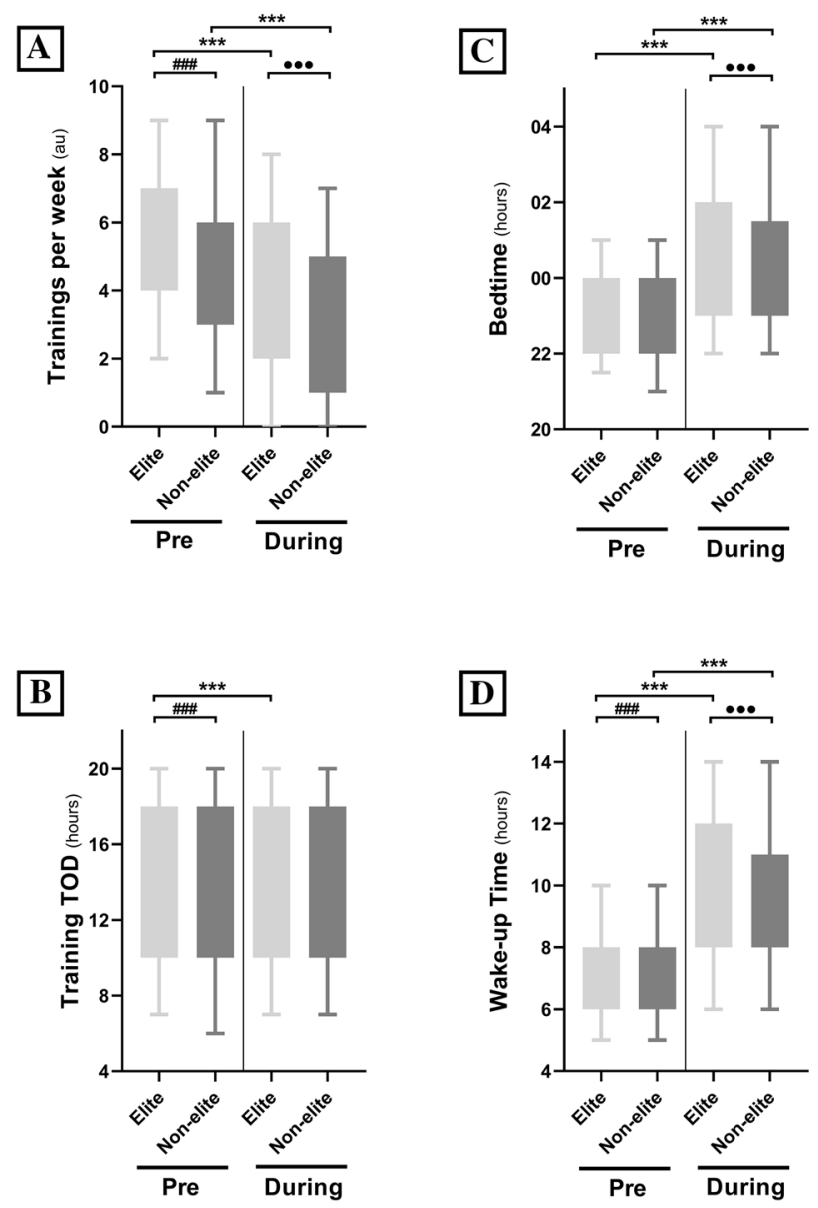

Fig. 5 Mean and 95\% confidence interval of sleep metrics and training habits pre- and during lockdown for elite athletes (light grey box; $n=1442$ ) and non-elite athletes (dark grey box; $n=2428$ ). A The number of training sessions per week, $\mathbf{B}$ the preferred time of day (TOD) to train, $\mathbf{C}$ bedtime, $\mathbf{D}$ wake-up time, $\mathbf{E}$ total sleep time (TST), $\mathbf{F}$ time in bed (TIB), $\mathbf{G}$ sleep-onset latency (SOL), and $\mathbf{H}$ sleep efficiency (SE). *, **, and $* * *$ mean significant effect of the lockdown

the effect of sleep-onset latency on PSQI and positively mediated the effect of sleep efficiency on PSQI scores. This suggests that athletes were compensating the longer sleeponset latency and the reduced sleep efficiency by longer total sleep time and time in bed. It therefore seems logical to recommend athletes target $9-10 \mathrm{~h}$ of sleep per night (in line with guidelines elsewhere [20,21, 24]), sleeping earlier (than what is observed during lockdown) and waking up later (compared with that observed pre-lockdown) [20, 21, 24].

However, despite a lockdown-mediated increased sleep duration, poorer sleep quality and a higher risk for insomnia were evident during lockdown, including lower sleep efficiency and greater difficulties falling and/or staying asleep. These are likely attributable to the altered homeostatic and circadian cues/processes [14], evidenced by surveyed athletes altering their normal routines (e.g., going to bed,
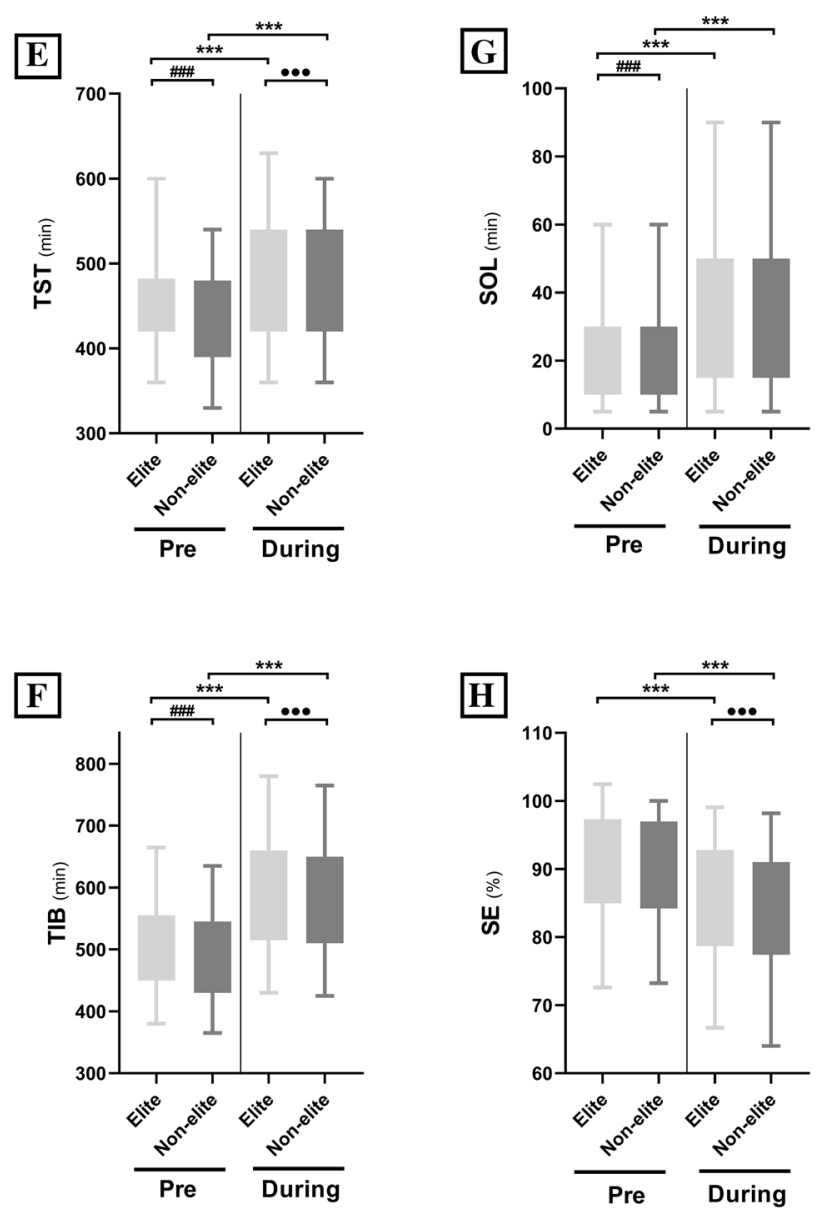

within groups at $p<0.05, p<0.01$, and $p<0.001$, respectively. Black dots $\cdot, \cdot$, and $\cdots$ mean difference between groups during lockdown at $p<0.05, p<0.01$, and $p<0.001$, respectively. \#, \#\#, and \#\#\# mean difference between groups pre-lockdown at $p<0.05, p<0.01$, and $p<0.001$, respectively. Significance is assessed by the two-way analysis of variance and the Bonferroni post-hoc test

waking up, and training later during lockdown). Later bedtimes were associated directly and indirectly with a lower sleep quality and higher insomnia. Here, it appears that 'late sleepers' are 'bad sleepers' with poorer sleep quality, more nightmares, and worse insomnia symptoms than their 'earlier sleepers' peers [34]. Adherence to regular sleep-wake schedule promotes optimal sleep propensity and consolidation [35], which will potentially promote health and maximize physical and cognitive performance [24].

\subsection{Eating Behavior}

Given meals are a potent zeitgeber within circadian regulation, it was interesting to see an increased likelihood to eat after midnight being associated directly with higher insomnia (Fig. 1) and indirectly through mediating the effects of sleep-onset latency and bedtimes on ISI scores (Fig. 3). 

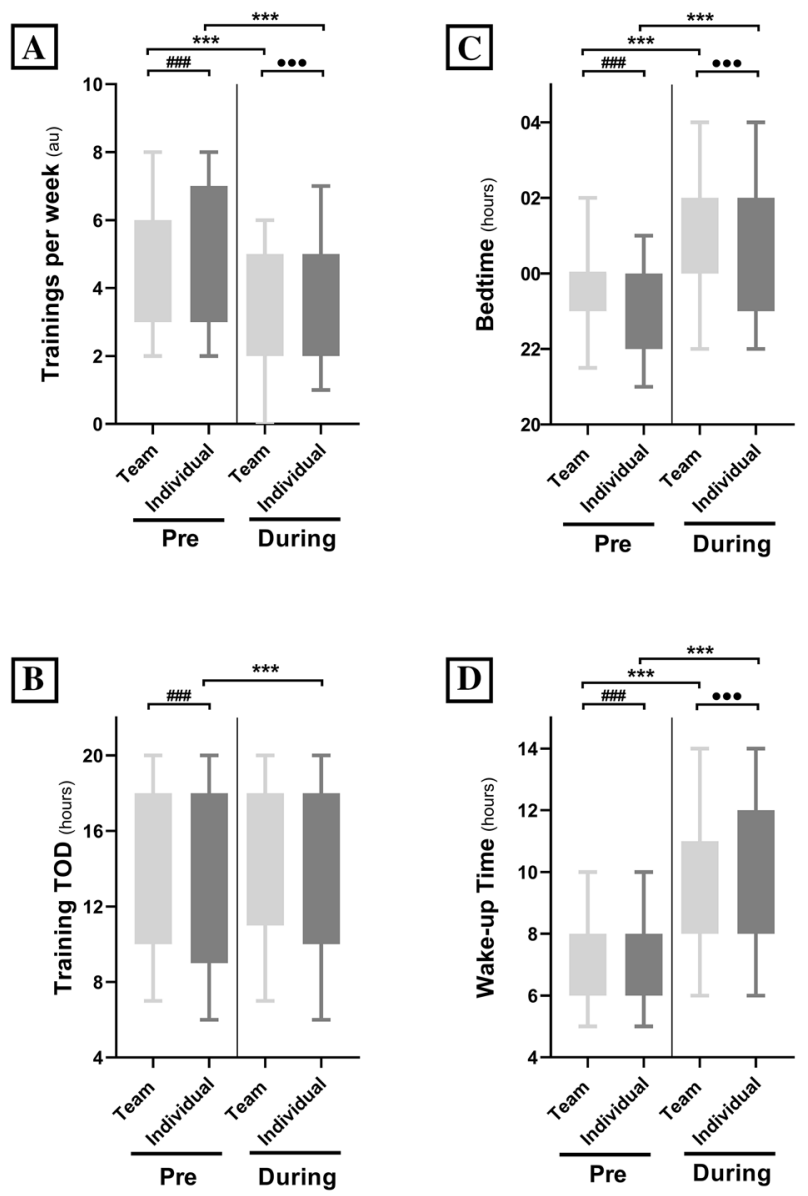

Fig. 6 Mean and 95\% confidence interval of sleep metrics and training habits pre- and during lockdown for individual-sport (light grey box; $n=1443$ ) and team-sport (dark grey box; $n=2427$ ) athletes. A The number of training sessions per week, $\mathbf{B}$ the preferred time of day to train (TOD), $\mathbf{C}$ bedtime, $\mathbf{D}$ wake-up time, $\mathbf{E}$ total sleep time (TST), $\mathbf{F}$ time in bed (TIB), G sleep-onset latency (SOL), and $\mathbf{H}$ sleep efficiency $(\mathrm{SE}) . *, * *$, and $* * *$ mean significant effect of the

Further, the change in body mass was directly associated with the change in PSQI scores, and indirectly associated with ISI scores through delaying bedtime. Late bedtimes create an imbalance of hunger, satiety, and metabolic hormones [25], and increase the intake of energy-dense nutrient-poor foods [36]. Indeed, late sleepers consumed more calories per day ( 248) compared with normal sleepers, associated with a higher body mass index in late sleepers [36]. Additionally, core body temperature and cortisol levels increase with food intake promoting alertness [37], indicative of the longer sleep-onset latency and later bedtimes observed in the current study. Therefore, it is recommended to not consume a heavy meal with undesirable nutritional content around bedtime. However, a light tryptophan-rich meal is encouraged $1 \mathrm{~h}$ prior to bedtime to allow proper digestion (e.g., milk and derivates, fish, white of eggs, red and white meat, soybean, sesame, and pumpkin seed).
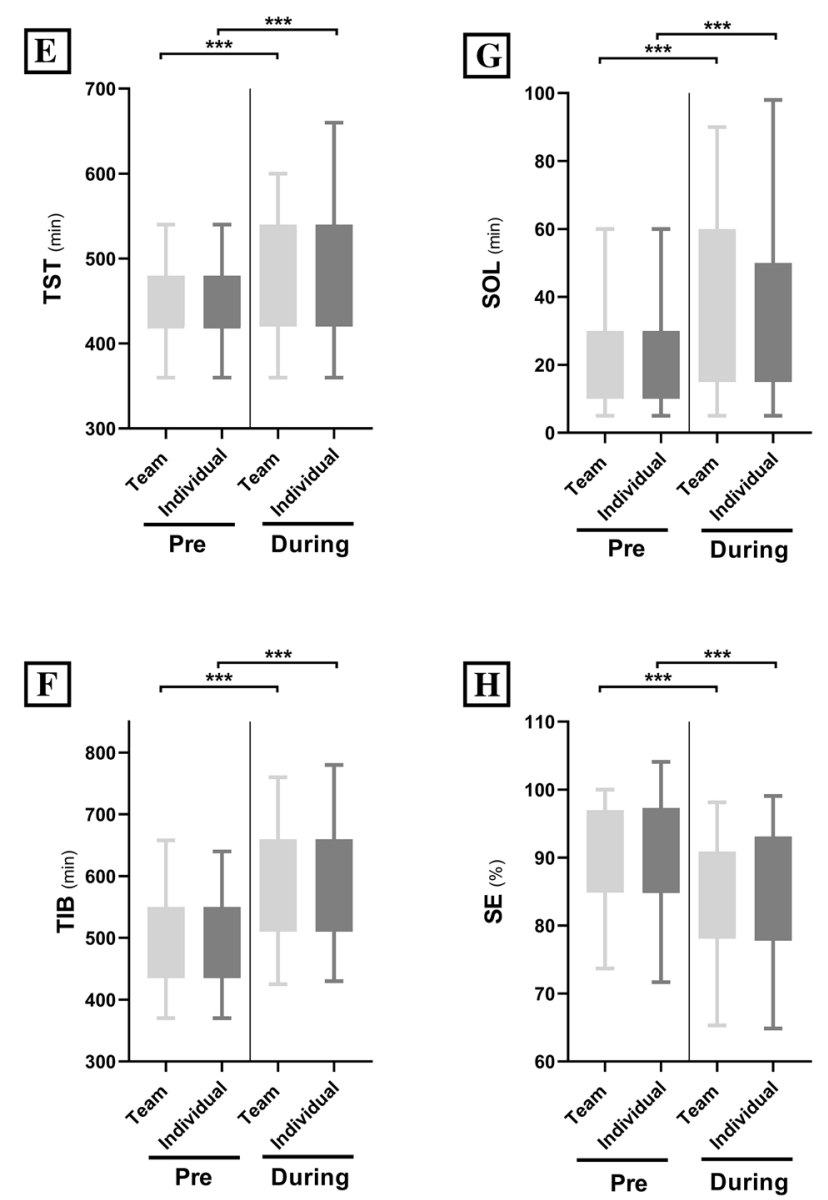

lockdown within groups at $p<0.05, p<0.01$, and $p<0.001$, respectively. $\cdot, \cdot$, and $\cdots$ mean difference between groups during lockdown at $p<0.05, p<0.01$, and $p<0.001$, respectively. \#, \#\#, and \#\#\# mean difference between groups pre-lockdown at $p<0.05, p<0.01$, and $p<0.001$, respectively. Significance is assessed by the two-way analysis of variance and the Bonferroni post-hoc test

\subsection{Smoking, Alcohol, and Caffeine}

The increased tobacco, caffeine, and alcohol consumption during lockdown: (i) influenced directly PSQI and ISI scores and (ii) mediated the effect of SOL on PSQI and ISI. Smoking [38] and alcohol consumption [39] are associated with a disturbed and undesirable sleep structure, including: (i) more non-rapid eye movement stage 1 (N1) sleep; and (ii) less slow-wave sleep. Similarly, caffeine consumption delays sleep-onset latency, decreases slow-wave sleep, and increases the amount of N2 [40], even when taken in small amounts [35]. Caffeine taken $6 \mathrm{~h}$ before bedtime can produce disruptive effects on sleep [40], given its long half-life (5-7 h) [41]. This tripartite ensemble of smoking, alcohol, and caffeine are modifiable behavioral factors open to intervention to acquiesce the COVID19-induced undesirable sleep effects. Specifically, athletes are recommended to 

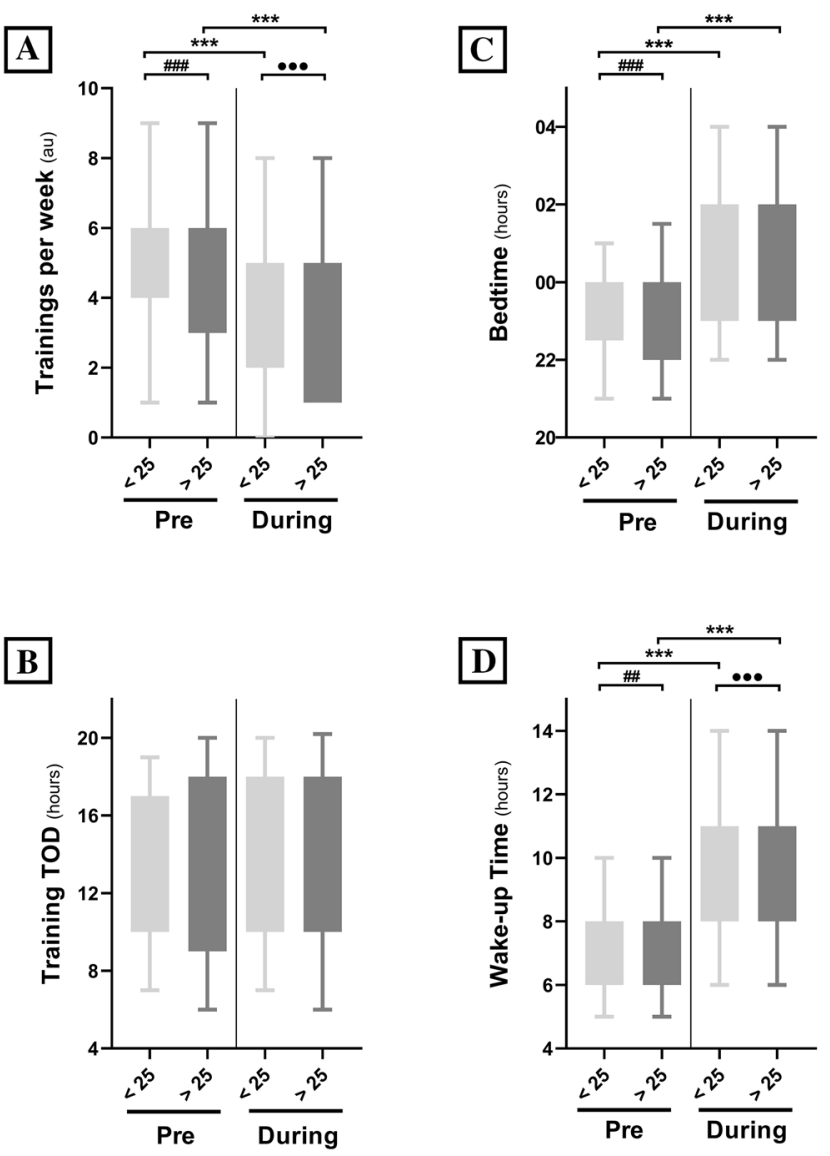

Fig. 7 Mean and 95\% confidence interval of sleep metrics and training habits pre- and during lockdown for age $\leq 25$ years (light grey box; $n=2595$ ) and age $>25$ years (dark grey box; $n=1275$ ) athletes. A The number of training sessions per week, $\mathbf{B}$ the preferred time of day (TOD) to train, $\mathbf{C}$ bedtime, $\mathbf{D}$ wake-up time, $\mathbf{E}$ total sleep time (TST), $\mathbf{F}$ time in bed (TIB), $\mathbf{G}$ sleep-onset latency (SOL), and $\mathbf{H}$ sleep efficiency $(\mathrm{SE}) . * * *$, and $* * *$ mean significant effect of the

avoid alcohol and cigarette consumption and not consume caffeine in the second half of the day to enhance deep sleep, which is more recuperative.

\subsection{Effect of Competitive Level}

Elite athletes slept longer during $(+14 \mathrm{~min})$ and pre(+18 min) lockdown compared with non-elite athletes $(p<0.001)$, as well as reporting a greater sleep efficiency during lockdown $(85 \pm 10 \%$ vs $83 \pm 10 \% ; p<0.001)$. Similarly, it has been reported that young elite athletes (16.5 years) show greater sleep quality compared with agematched non-elite athletes and non-athletes [42]. In our sample, elite athletes showed a greater lockdown-induced circadian misalignment compared with non-elite athletes. Additionally, elite athletes reported higher lockdownmediated insomnia $(+52 \%$ vs $+45 \%)$ and lower sleep quality $(+42 \% \mathrm{vs}+30 \%)$. This is likely linked to the reduced
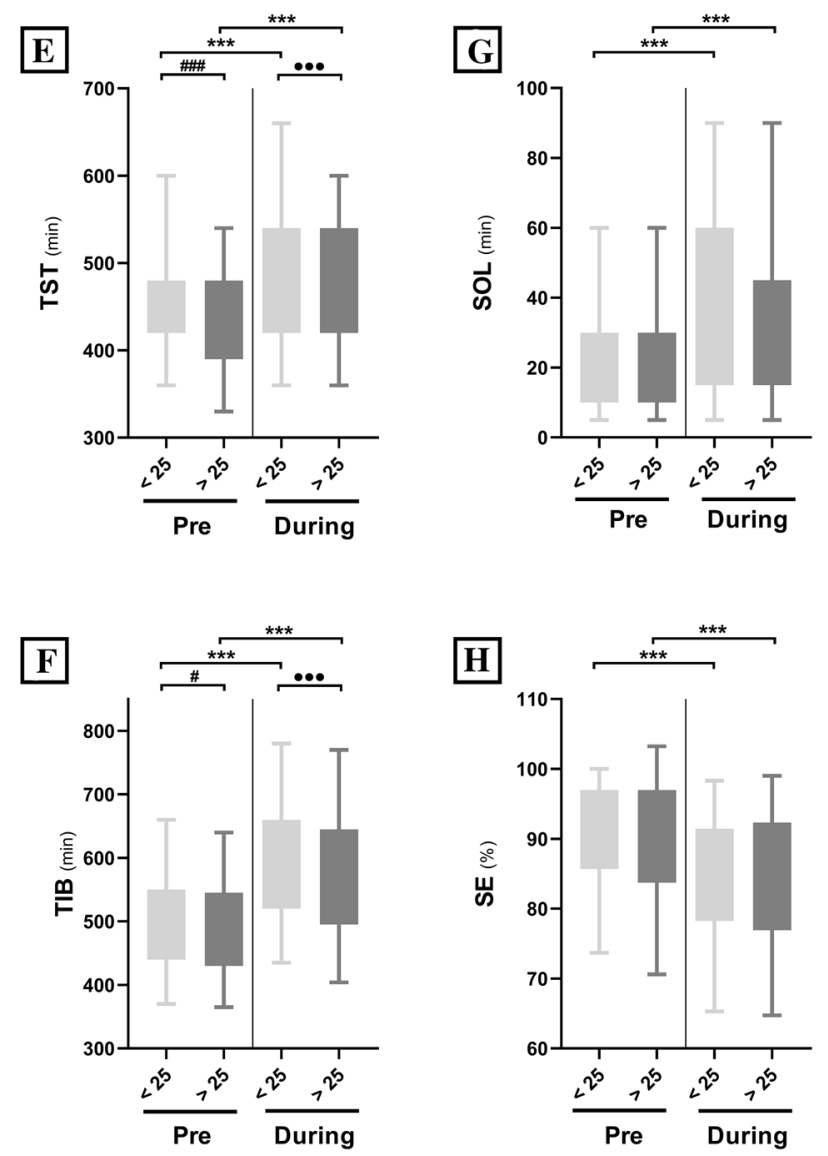

lockdown within groups at $p<0.05, p<0.01$, and $p<0.001$, respectively. $\cdot, \cdot$, and $\cdots$ mean difference between groups during lockdown at $p<0.05, p<0.01$, and $p<0.001$, respectively. \#, \#, and \#\# meas difference between groups pre-lockdown at $p<0.05, p<0.01$, and $p<0.001$, respectively. Significance is assessed by the two-way analysis of variance and the Bonferroni post-hoc test

weekly training sessions and circadian/homeostatic misalignment due to lockdown, conspiring to reduce sleep drive and quality. The reduced number of training sessions per week during lockdown was associated with higher ISI scores. Indeed, it has been reported that regular and acute physical exercise could enhance total sleep time and sleep efficiency and reduce sleep-onset latency $[35,41]$.

\subsection{Effect of Sport Discipline}

Pre-lockdown, individual-sport athletes slept (-56 min) and woke up ( $-18 \mathrm{~min}$ ) earlier compared with team-sport athletes, as shown elsewhere [43]. However, during lockdown, individual athletes showed the greatest shift towards 'eveningness' (i.e., greatest circadian delay) with later bed $(+7 \%$ vs $+2 \%)$ and wake-up (+40\% vs $+31 \%)$ times and a later preferred TOD to train $(+6.5 \%$ vs $+0.5 \%)$ compared with team-sport athletes. This finding is consistent with the 

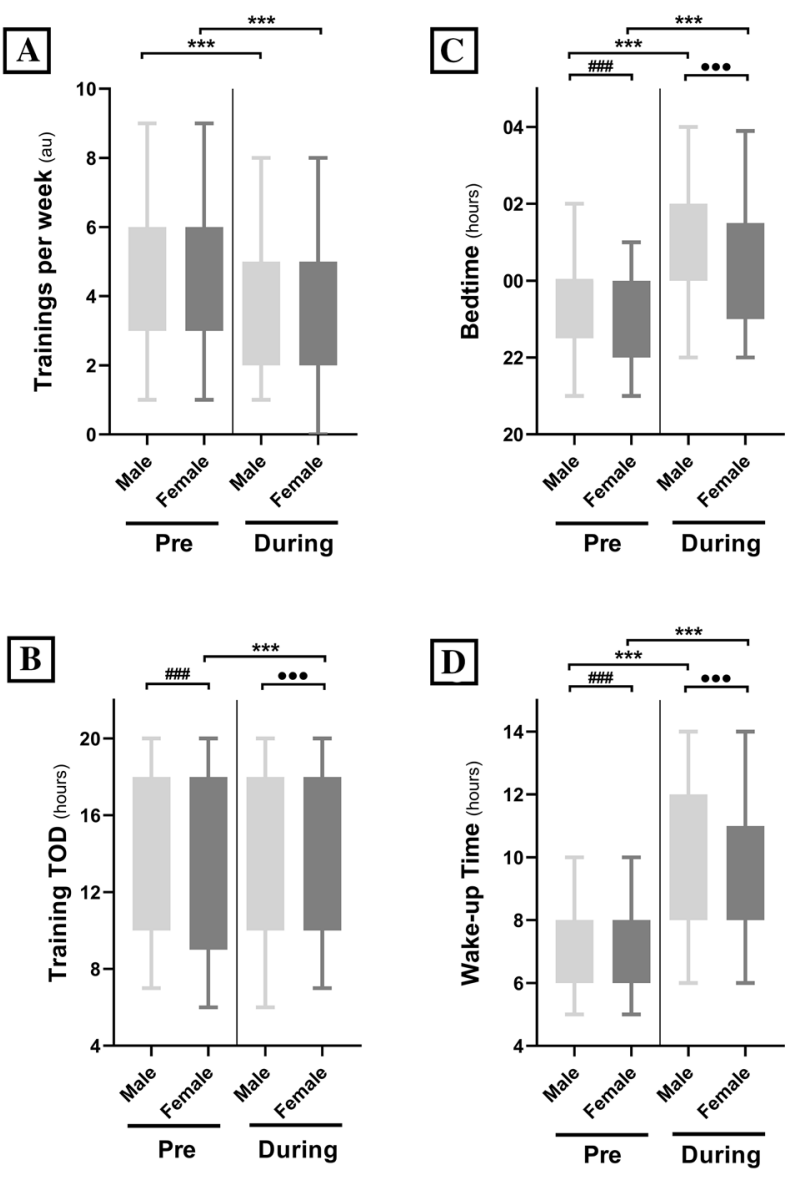

Fig. 8 Mean and 95\% confidence interval of sleep metrics and training habits pre- and during lockdown for male athletes (light grey box; $n=2106$ ) and female (dark grey box; $n=1764$ ) athletes. A The number of training sessions per week, $\mathbf{B}$ the preferred time of day (TOD) to train, $\mathbf{C}$ bedtime, $\mathbf{D}$ wake-up time, $\mathbf{E}$ total sleep time (TST), $\mathbf{F}$ time in bed (TIB), G sleep-onset latency (SOL), and $\mathbf{H}$ sleep efficiency (SE). *, **, and *** mean significant effect of the lockdown

shift of activity levels concomitantly with the change in bedtime and wake-up preferences [34, 44]. During lockdown, better sleep quality was associated with exercising earlier, but not later during the day [45]. Therefore, outdoor exercise and light exposure early during the day could better align the body clock to desirable sleep-focused circadian rhythms, thus promoting sleep at a preferential circadian phase [45].

\subsection{Effect of Age}

Training frequency and sleep quality of older athletes evidenced greater lockdown-mediated disruption compared with younger athletes, respectively. Younger athletes reported smaller increases in PSQI $(+31 \%$ vs $+47 \%)$ and ISI (+ $+43 \%$ vs $+65 \%)$ scores compared with older athletes, indicating a greater resilience to lockdown-mediated changes; in support of existing albeit limited data in the general population [9]. Indeed, young adults are less disturbed by circadian
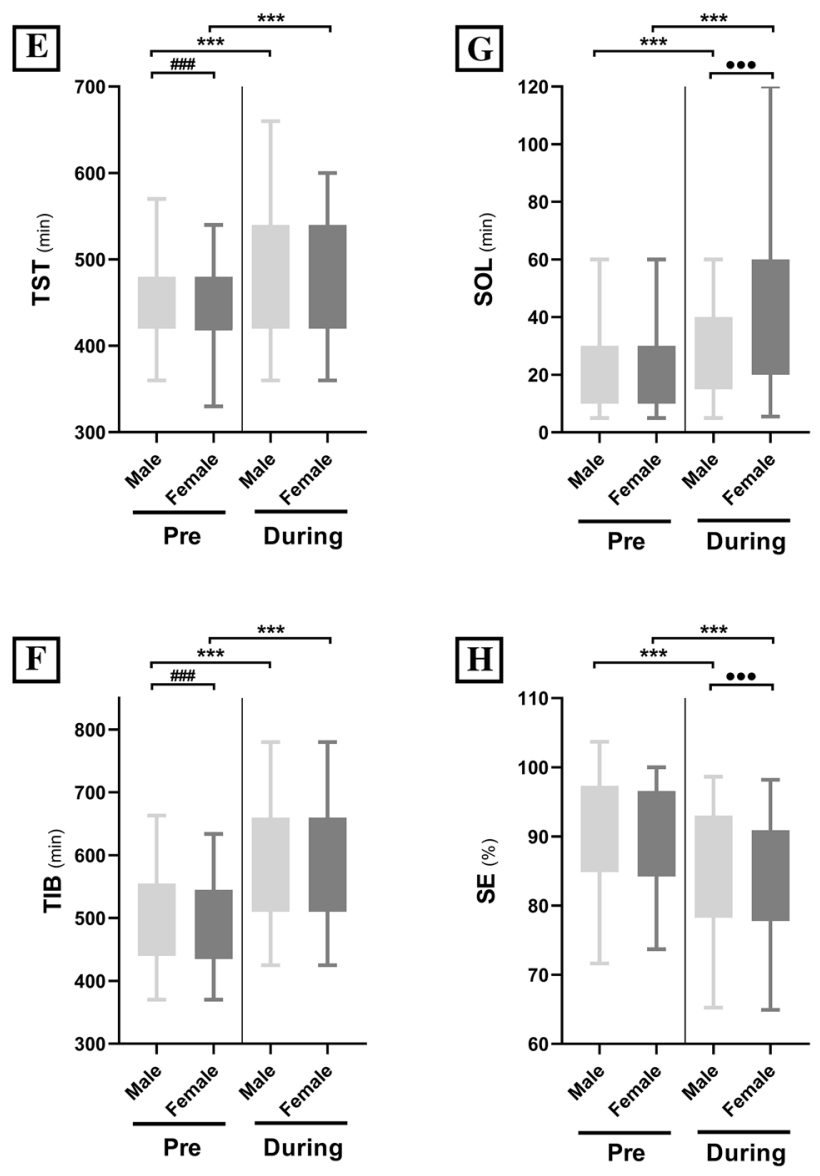

within groups at $p<0.05, p<0.01$, and $p<0.001$, respectively. $\cdot, \cdot$, and $\cdots$ mean difference between groups during lockdown at $p<0.05$,

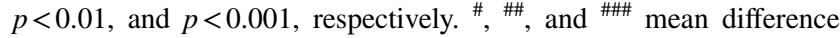
between groups pre-lockdown at $p<0.05, p<0.01$, and $p<0.001$, respectively. Significance is assessed by the two-way analysis of variance and the Bonferroni post-hoc test

misalignment (e.g., jet-lag) than older adults [9]. Napping duration $(+124 \%)$ and frequency $(+55 \%)$ increased in the older group, which may have been a response to increased insomnia symptoms and lowered nocturnal sleep quality in the older athlete during lockdown. Longer and later daytime napping affected ISI scores directly and indirectly by means of longer sleep-onset latency and later bedtimes. Although long naps are efficient in enhancing performance after partial sleep deprivation [46], they can increase daytime sleepiness and reduce subsequent performance compared with short naps after appropriate nocturnal sleep [47]. Taking long (>90 min), or late (>16:00) diurnal naps decreases the homeostatic drive for sleep [35] and increase insomnia symptoms during the subsequent night. Preferably, a moderate dose of caffeine immediately prior to starting a 30-min midday nap to maximize alertness and avoid sleep inertia at the awakening [48-50], would appear prudent to not interfere with the upcoming nocturnal sleep. 


\subsection{Effect of Sex}

Sex differences were also evident within the present data, although independent of the effects of lockdown. In general, female athletes had less desirable sleep quality parameters (e.g., higher PSQI) and greater insomnia-like symptoms (e.g., higher ISI) than male athletes, as seen elsewhere [51]. Although female athletes reported higher PSQI both pre- and during lockdown compared with male athletes, the lockdown-mediated reduced sleep quality was not different between groups (female athletes: $+34.81 \%$ vs male athletes: $+35.27 \%$ ). Similarly, female athletes showed a higher prevalence of anxiety, depression, and sleep disorder compared with male athletes [51]. These results confirm the sex-based differences in sleep characteristics reported elsewhere and that they endure despite the pandemic [52]. Finally, female athletes preferred to train at an earlier TOD compared with male athletes pre- $(-53 \mathrm{~min})$ and during (-37 min) lockdown, this might be due to having an earlier circadian phase of melatonin and core body temperature compared with male athletes [53].

\subsection{Strengths and Limitations}

The main strength of this study is its large sample size (largest survey to date on sleep in athletes). This international sample presents a wide range of sport disciplines across sexes. The survey and recommendations were developed by an international research team from diverse background and cultures, promoting their applications. Conversely, the current study presents some limitations. The main limitation of the present study is that we had to collect data about the pre-lockdown period (and during lockdown for some participants) by a retrospective self-report, which could be subject to recall bias. The current study was advertised online and it could be subject to sampling bias (e.g., non-athlete claiming that they are athletes or non-elite athletes claiming that they are elite athletes; maliciously or otherwise). Additionally, the lockdown level (e.g., partial or total lockdown) was not the same across the world at the time of survey, which could limit the current conclusions to some extent. Additionally, linking sleep quality to psychometric measures such as stress and mood would have been more informative about athletes' wellness during lockdown. The data were not explored relative to athlete chronotype [44], as this was not established within the sample utilized. The data see very large standard deviations, indicative of high inter-individual variability within the sample. Indeed, individual preferences should be prioritized when developing sleep hygiene protocols rather than a standardized 'one size fits all' intervention [21]. Further, whether adolescent population-specific recommendations and practice are warranted requires further thought [54]. As a minimal number of adolescents were included within this sample, there was insufficient statistical power to model adolescents as a further sub-group to the employed younger and older sub-groups. Additionally, when, how and if sleep medications (including melatonin, Circadian ${ }^{\circledR}$ ) can be used within periods of lockdown require careful consideration [55] and are beyond the scope of the current article.

\subsection{Recommendations for Future 'Lockdown-Like' Restrictions}

- Adherence to sleep hygiene could protect, at least in part, against the observed lower sleep quality and higher insomnia in athletes. Helping protect athlete physical and mental health, limit detraining and facilitate a return to training and competition upon lockdown easing.

- It is recommended to avoid long and late napping whilst opting for a moderate dose of caffeine prior to a 30 -min post-lunch nap.

- Avoid alcohol and cigarette consumption, and caffeine in the second half of the day (it would seem prudent to abstain from these if possible/viable).

- Maintain regular schedules of meals (including appropriate composition), and bed and wake-up times.

- Avoid heavy meals near bedtime and opt for a light tryptophan-rich snack $1 \mathrm{~h}$ pre-bedtime.

- Outdoor training sessions, early in the day, are highly recommended during lockdown.

\section{Conclusions}

From the largest survey to date on sleep in athletes (irrespective of COVID-19), the COVID-19-mediated lockdown phase delayed athlete's circadian rhythms, reduced sleep quality, and increased insomnia-like symptoms to a greater magnitude than shown in the general public [5]. Lockdowninduced behavioral changes (some of them modifiable) lowered sleep quality and increased insomnia in athletes. Directly, these were longer sleep-onset latency and later bedtimes and indirectly by way of nocturnal eating behaviors, longer and later napping, and higher caffeine, alcohol, and cigarette consumption. Moreover, the preferred TOD to train seems to have a greater impact on sleep quality and insomnia than the number of training sessions per week. We also highlight the vulnerability of elite, individual-sport, and older athletes to these lockdown-induced changes. Further data establishing whether the demonstrated lockdownmediated circadian misalignment is transient or enduring appear prudent.

Supplementary Information The online version contains supplementary material available at https://doi.org/10.1007/s40279-021-01601-y. 


\section{Declarations}

Funding No funding related to the project has been received.

Conflicts of interest/Competing interests All authors declare that there is no conflict of interest to disclose.

Ethics approval The current study was approved by Farhat Hached Hospital, Sousse, Tunisia (IRB\# FH020720).

Consent to participate All respondents provided informed consent before participating in the survey.

Consent for publication All respondents provided consent for anonymous data use for research purposes and publications.

Availability of data and material All data are stored on institutional servers of the corresponding author and available on reasonable request.

Code availability Not applicable.

Author contributions The study was designed by MR, KT, HC, and $\mathrm{KC}$. All authors have been involved in the survey development and translation and data collection. MR performed the statistical analysis and prepared the first draft. The manuscript was critically revised by DER, AA, HF, JV, MN, HBS, TD, JAW, LT, and KC. All authors approved of the final version to be published and agree to be accountable for any part of the work.

\section{References}

1. Ammar A, Trabelsi K, Brach M, et al. Effects of home confinement on mental health and lifestyle behaviours during the COVID-19 outbreak: insights from the ECLB-COVID19 multicentre study. Biol Sport. 2021;38:9-21. https://doi.org/10.5114/ biolsport.2020.96857.

2. Chirico A, Lucidi F, Galli F, et al. COVID-19 outbreak and physical activity in the Italian population: a cross-sectional analysis of the underlying psychosocial mechanisms. Front Psychol. 2020;11:1-13. https://doi.org/10.3389/fpsyg.2020.02100.

3. Ammar A, Chtourou H, Boukhris O, et al. Covid-19 home confinement negatively impacts social participation and life satisfaction: a worldwide multicenter study. Int J Environ Res Public Health. 2020;17:1-17. https://doi.org/10.3390/ijerph17176237.

4. Stanton R, To QG, Khalesi S, et al. Depression, anxiety and stress during COVID-19: associations with changes in physical activity, sleep, tobacco and alcohol use in Australian adults. Int J Environ Res Public Health. 2020;17:1-13. https://doi.org/10.3390/ijerp h17114065.

5. Trabelsi K, Ammar A, Liwa M, et al. Globally altered sleep patterns and physical activity levels by confinement in 5056 individuals: ECLB COVID-19 international online survey. Biol Sport. 2021;38:495-506. https://doi.org/10.5114/biolsport.2021.101605.

6. Facer-Childs ER, Hoffman D, Tran JN, et al. Sleep and mental health in athletes during COVID-19 lockdown. Sleep. 2021;44(5):zsaa261. https://doi.org/10.1093/sleep/zsaa261.

7. Pillay L, Janse van Rensburg DCC, Jansen van Rensburg A, Nowhere to hide: the significant impact of coronavirus disease, et al. (COVID-19) measures on elite and semi-elite South African athletes. J Sci Med Sport. 2019;2020(23):670-9. https://doi.org/ 10.1016/j.jsams.2020.05.016.
8. Beck F, Léger D, Fressard L, et al. Covid-19 health crisis and lockdown associated with high level of sleep complaints and hypnotic uptake at the population level. J Sleep Res. 2020;30(1): 313119. https://doi.org/10.1111/jsr.13119.

9. Hood S, Amir S. The aging clock: circadian rhythms and later life. J Clin Invest. 2017;127:437-46. https://doi.org/10.1172/JCI90 328.

10. Borbély AA, Daan S, Wirz-Justice A, et al. The two-process model of sleep regulation: a reappraisal. J Sleep Res. 2016;25:131-43. https://doi.org/10.1111/jsr.12371.

11. Taylor SR, Rogers GG, Driver HS. Effects of training volume on sleep, psychological, and selected physiological profiles of elite female swimmers. Med Sci Sports Exerc. 1997;29:688-93. https:// doi.org/10.1097/00005768-199705000-00016.

12. Brand S, Beck J, Gerber M, et al. Evidence of favorable sleep-EEG patterns in adolescent male vigorous football players compared to controls. World J Biol Psychiatry. 2010;11:465-75. https://doi. org/10.3109/15622970903079820.

13. Youngstedt SD. Effects of exercise on sleep. Clin Sports Med. 2005;24:355-65. https://doi.org/10.1016/j.csm.2004.12.003.

14. Davenne D. Sleep of athletes: problems and possible solutions. Biol Rhythm Res. 2009;40:45-52. https://doi.org/10.1080/09291 010802067023.

15. Romdhani M, Hammouda O, Chaabouni Y, et al. Sleep deprivation affects post-lunch dip performances, biomarkers of muscle damage and antioxidant status. Biol Sport. 2019;36:55-65. https:// doi.org/10.5114/biolsport.2018.78907.

16. Romdhani M, Hammouda O, Smari K, et al. Total sleep deprivation and recovery sleep affect the diurnal variation of agility performance: the gender differences. J Strength Cond Res. 2021;35:132-40. https://doi.org/10.1519/jsc.0000000000002614.

17. Thomas JM, Kern PA, Bush HM, et al. Circadian rhythm phase shifts caused by timed exercise vary with chronotype. JCI Insight. 2020;5:12-4. https://doi.org/10.1172/jci.insight.134270.

18. Bonato M, la Torre A, Saresella M, et al. Effect of high-intensity interval training versus small-sided games training on sleep and salivary cortisol level. Int J Sports Physiol Perform. 2020;15:1237-44. https://doi.org/10.1123/IJSPP.2019-0498.

19. Halson SL. Stealing sleep: is sport or society to blame? Br J Sports Med. 2016;50:381. https://doi.org/10.1136/bjsports-2015-094961.

20. Roberts SSH, Teo WP, Warmington SA. Effects of training and competition on the sleep of elite athletes: a systematic review and meta-analysis. Br J Sports Med. 2019;53:513-22. https://doi.org/ 10.1136/bjsports-2018-099322.

21. Walsh NP, Halson SL, Sargent C, et al. Sleep and the athlete : narrative review and 2021 expert consensus recommendations. Br J Sports Med. 2021;55:1-13. https://doi.org/10.1136/bjspo rts-2020-102025.

22. Gupta L, Morgan K, Gilchrist S. Does elite sport degrade sleep quality? A systematic review. Sport Med. 2017;47:1317-33. https://doi.org/10.1007/s40279-016-0650-6.

23. Watson NF, Badr MS, Belenky G, et al. Joint consensus statement of the American Academy of Sleep Medicine and Sleep Research Society on the recommended amount of sleep for a healthy adult: methodology and discussion. J Clin Sleep Med. 2015;11:931-52. https://doi.org/10.5664/jcsm.4950.

24. Nédélec M, Halson S, Delecroix B, et al. Sleep hygiene and recovery strategies in elite soccer players. Sport Med. 2015;45:154759. https://doi.org/10.1007/s40279-015-0377-9.

25. Spiegel K, Tasali E, Penev P, et al. Brief communication: sleep curtailment in healthy young men is associated with decreased leptin levels, elevated ghrelin levels, and increased hunger and appetite. Ann Intern Med. 2004;141:846-50. https://doi.org/10. 7326/0003-4819-141-11-200412070-00008.

26. Yousfi N, Bragazzi NL, Briki W, et al. The COVID-19 pandemic: how to maintain a healthy immune system during the lockdown. 
A multidisciplinary approach with special focus on athletes. Biol Sport. 2020;37:211-6. https://doi.org/10.5114/biolsport.2020. 95125.

27. Whitley E, Ball J. Statistics review 4: sample size calculations. Crit Care. 2002;6(4):3351-441. https://doi.org/10.1186/CC1521.

28. World Health Organization (WHO). Adolescent and young adult health. 2021. https://www.who.int/news-room/fact-sheets/detail/ adolescents-health-risks-and-solutions. Accessed 24 Jul 2021.

29. Buysse DJ, Reynolds CF, Monk TH, et al. The Pittsburgh Sleep Quality Index: a new instrument for psychiatric practice and research. Psychiatry Res. 1989;28:193-213. https://doi.org/10. 1016/0165-1781(89)90047-4.

30. Bastien $\mathrm{CH}$, Vallières $\mathrm{A}$, Morin $\mathrm{CM}$. Validation of the Insomnia Severity Index as an outcome measure for insomnia research. Sleep Med. 2001;2:297-307. https://doi.org/10.1016/S13899457(00)00065-4.

31. Cohen J. A power primer. Psychol Bull. 1992;112:155-9. https:// doi.org/10.1037/0033-2909.112.1.155.

32. Hayes AF, Scharkow M. The relative trustworthiness of inferential tests of the indirect effect in statistical mediation analysis: does method really matter? Psychol Sci. 2013;24:1918-27. https://doi. org/10.1177/0956797613480187.

33. Vlahoyiannis A, Aphamis G, Bogdanis GC, et al. Deconstructing athletes' sleep: a systematic review of the influence of age, sex, athletic expertise, sport type, and season on sleep characteristics. J Sport Health Sci. 2021;10(4):387-402. https://doi.org/10.1016/j. jshs.2020.03.006.

34. Merikanto I, Kronholm E, Peltonen M, et al. Relation of chronotype to sleep complaints in the general finnish population. Chronobiol Int. 2012;29:311-7. https://doi.org/10.3109/07420528.2012. 655870 .

35. Stepanski EJ, Wyatt JK. Use of sleep hygiene in the treatment of insomnia. Sleep Med Rev. 2003;7:215-25. https://doi.org/10. 1053/smrv.2001.0246.

36. Baron KG, Reid KJ, Kern AS, et al. Role of sleep timing in caloric intake and BMI. Obesity. 2011;19:1374-81. https://doi.org/10. 1038/oby.2011.100.

37. Golem DL, Martin-Biggers JT, Koenings MM, et al. An integrative review of sleep for nutrition professionals. Adv Nutr. 2014;5:742-59. https://doi.org/10.3945/an.114.006809.

38. Zhang L, Samet J, Caffo B, et al. Cigarette smoking and nocturnal sleep architecture. Am J Epidemiol. 2006;164:529-37. https://doi. org/10.1093/aje/kwj231.

39. van Schrojenstein LM, Roth T, Roehrs T, et al. Alcohol hangover, sleep quality, and daytime sleepiness. Sleep Vigil. 2017;1:37-41. https://doi.org/10.1007/s41782-017-0008-7.

40. Christopher D, Timothy R, John S, et al. Caffeine effects on sleep taken 0, 3, or 6 hours before going to bed. J Clin Sleep Med. 2013;9:1195-200.

41. Altena E, Baglioni C, Espie CA, et al. Dealing with sleep problems during home confinement due to the COVID-19 outbreak: practical recommendations from a task force of the European CBT-I Academy. J Sleep Res. 2020;29:1-7. https://doi.org/10. 1111/jsr.13052.

42. Harris A, Gundersen H, Andreassen PM, et al. A comparative study of sleep and mood between young elite athletes and age-matched controls. J Phys Act Health. 2017;14:465-73. https:// doi.org/10.1123/jpah.2016-0513.

43. Lastella M, Roach GD, Halson SL, et al. Sleep/wake behaviours of elite athletes from individual and team sports. Eur J Sport Sci. 2015;15:94-100. https://doi.org/10.1080/17461391.2014.932016.

44. Vitale JA, Roveda E, Montaruli A, et al. Chronotype influences activity circadian rhythm and sleep: differences in sleep quality between weekdays and weekend. Chronobiol Int. 2015;32:405-15. https://doi.org/10.3109/07420528.2014.986273.

45. Carrigan N, Wearn A, Meky S, et al. Sleep quality, mental health and circadian rhythms during COVID lockdown: results from the SleepQuest Study. medRxiv. 2020. https://doi.org/10.1101/2020. 07.08.20148171.

46. Romdhani M, Souissi N, Chaabouni Y, et al. Improved physical performance and decreased muscular and oxidative damage with postlunch napping after partial sleep deprivation in Athletes. Int $\mathbf{J}$ Sports Physiol Perform. 2020;15:874-83. https://doi.org/10.1123/ ijspp.2019-0308.

47. Romdhani M, Dergaa I, Moussa-Chamari I, et al. The effect of post-lunch napping on mood, reaction time and antioxidant defense during repeated sprint exercise. Biol Sport. 2021;38:62938. https://doi.org/10.5114/biolsport.2021.103569.

48. Romdhani M, Souissi N, Moussa-Chamari I, et al. Caffeine use or napping to enhance repeated sprint performance after partial sleep deprivation: why not both? Int J Sports Physiol Perform. 2021;16:711-8. https://doi.org/10.1123/ijspp.2019-0792.

49. Romdhani M, Souissi N, Dergaa I, et al. The effect of experimental recuperative and appetitive post-lunch nap opportunities, with or without caffeine, on mood and reaction time in highly trained athletes. Front Psychol. 2021;12:1-11. https://doi.org/10.3389/ fpsyg.2021.720493.

50. Souabni M, Hammouda O, Romdhani M, et al. Beneits of daytime napping opportunity on physical and cognitive performances in physically active participants: a systematic review. Sport Med. 2021. https://doi.org/10.1007/s40279-021-01482-1 (in press).

51. Schaal K, Tafflet M, Nassif H, et al. Psychological balance in high level athletes: gender-Based differences and sport-specific patterns. PLoS ONE. 2011;6: e19007. https://doi.org/10.1371/journ al.pone.0019007.

52. Leeder J, Glaister M, Pizzoferro K, et al. Sleep duration and quality in elite athletes measured using wristwatch actigraphy. J Sports Sci. 2012;30:541-5. https://doi.org/10.1080/02640414. 2012.660188.

53. Cain SW, Dennison CF, Zeitzer JM, et al. Sex differences in phase angle of entrainment and melatonin amplitude in humans. J Biol Rhythms. 2010;25:288-96. https://doi.org/10.1177/0748730410 374943.

54. Taylor L, Chrismas BCR, Dascombe B, et al. The importance of monitoring sleep within adolescent athletes: athletic, academic, and health considerations. Front Physiol. 2016;7:1-6. https://doi. org/10.3389/fphys.2016.00101.

55. Taylor L, Chrismas BCR, Dascombe B, et al. Sleep medication and athletic performance: the evidence for practitioners and future research directions. Front Physiol. 2016;7:1-5. https://doi.org/10. 3389/fphys.2016.00083.

\title{
Authors and Affiliations
}

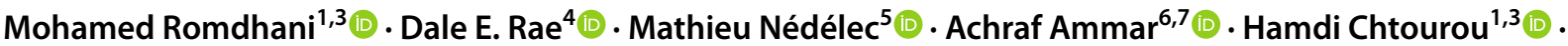

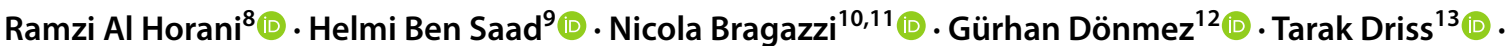

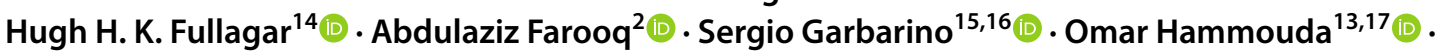 \\ Bahar Hassanmirzaei ${ }^{18,19} \cdot$ Karim Khalladi $^{2}$ (D) Syrine Khemila ${ }^{3,20} \cdot$ Leonardo Jose Mataruna-Dos-Santos ${ }^{21,22}$.
}




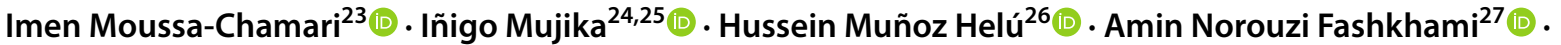
Laisa Liane Paineiras-Domingos ${ }^{28,29}$ (1) - Mehrshad Rahbari Khaneghah ${ }^{27}$ (i) - Yoshitomo Saita ${ }^{30}$ (1) Khaled Trabelsi ${ }^{1,31}$. Jacopo Antonino Vitale ${ }^{32}$. Jad Adrian Washif ${ }^{33}$. Jo . Johanna Weber ${ }^{34,35}$ (1) Nizar Souissi ${ }^{3,20} \cdot$ Lee Taylor $^{36,37}$ (1) Karim Chamari ${ }^{2,20}$

Mohamed Romdhani romdhaniroma@gmail.com

1 High Institute of Sport and Physical Education of Sfax, Sfax University, Sfax, Tunisia

2 Aspetar, Orthopaedic and Sports Medicine Hospital, FIFA Medical Center of Excellence, Doha, Qatar

3 Physical Activity, Sport and Health, UR18JS01, National Observatory of Sports, BP 143 Olympic City, 1003 Tunis, Tunisia

4 Division of Exercise Science and Sports Medicine, Department of Human Biology, Faculty of Health Sciences, University of Cape Town, Cape Town, South Africa

5 Laboratory Sport, Expertise and Performance (EA 7370), French National Institute of Sport (INSEP), Paris, France

6 Institute of Sport Science, Otto-Von-Guericke University, 39106 Magdeburg, Germany

7 Department of Training and Movement Science, Institute of Sport Science, Johannes Gutenberg-University Mainz, 55099 Mainz, Germany

8 Department of Exercise Science, Yarmouk University, Irbid, Jordan

9 Université de Sousse, Hôpital Farhat HACHED, Faculté de Médecine de Sousse, Laboratoire de Recherche (LR12SP09) "Insuffisance Cardiaque", Sousse, Tunisie

10 Department of Health Sciences, Postgraduate School of Public Health, University of Genoa, Genoa, Italy

11 Laboratory for Industrial and Applied Mathematics, Department of Mathematics and Statistics, York University, Toronto, ON, Canada

12 Department of Sports Medicine, Hacettepe University School of Medicine, Ankara, Turkey

13 Interdisciplinary Laboratory in Neurosciences, Physiology and Psychology: Physical Activity, Health and Learning (LINP2) UFR STAPS, UPL, Paris Nanterre University, Nanterre, France

14 School of Sport, Exercise and Rehabilitation, Faculty of Health, University of Technology Sydney, Sydney, NSW, Australia

15 Department of Neuroscience, Rehabilitation, Ophthalmology, Genetics and Maternal-Infantile Sciences, University of Genoa, Genoa, Italy

16 Post-Graduate School of Occupational Medicine, Università Cattolica del Sacro Cuore, Roma, Italy

17 Research Laboratory, Molecular Bases of Human Pathology, LR19ES13, Faculty of Medicine, University of Sfax, Sfax, Tunisia
18 Sports Medicine Research Center, Neuroscience Institute, Tehran University of Medical Sciences, Tehran, Iran

19 Iran Football Medical Assessment and Rehabilitation Center - IFMARC, FIFA Medical Center of Excellence, Tehran, Iran

20 High Institute of Sport and Physical Education, Ksar-Said Manouba University, Manouba, Tunisia

21 Department of Sport Management, Faculty of Management, Canadian University of Dubai, Dubai, United Arab Emirates

22 Centre for Trust, Peace and Social Relation, Coventry University, Coventry, UK

23 Physical Education Department, College of Education, Qatar University, Doha, Qatar

24 Department of Physiology, Faculty of Medicine and Nursing, University of the Basque Country, Leioa, Basque Country, Spain

25 Exercise Science Laboratory, Faculty of Medicine, School of Kinesiology, Universidad Finis Terrae, Santiago, Chile

26 Department of Economic-Administrative Sciences, Universidad Autónoma de Occidente, Los Mochis, Sinaloa, Mexico

27 Esteghlal Physiotherapy Clinic, EPC, Teheran, Iran

28 Programa de Pós-Graduação Em Ciências Médicas, Universidade do Estado do Rio de Janeiro, Rio de Janeiro, Brazil

29 Departamento de Fisioterapia, Instituto de Ciências da Saúde, Universidade Federal da Bahia, Salvador, Bahia, Brazil

30 Department of Sports and Regenerative Medicine, Juntendo University, Tokyo, Japan

31 Research Laboratory: Education, Motricity, Sport and Health, EM2S, LR19JS01, University of Sfax, Sfax, Tunisia

32 IRCCS Istituto Ortopedico Galeazzi, Milan, Italy

33 Sports Performance Division, National Sports Institute of Malaysia, Kuala Lumpur, Malaysia

34 Neurocognition and Action, Biomechanics, Bielefeld University, Bielefeld, Germany

35 Institute for Sports Science, Christian-Albrechts-University of Kiel, Kiel, Germany

36 School of Sport, Exercise and Health Sciences, National Centre for Sport and Exercise Medicine (NCSEM), Loughborough University, Loughborough, UK

37 Sport and Exercise Discipline Group, Faculty of Health, University of Technology Sydney (UTS), Sydney, NSW, Australia 